\title{
Lagrangian coherent structures in tropical cyclone intensification
}

\author{
B. Rutherford ${ }^{1}$, G. Dangelmayr ${ }^{2}$, and M. T. Montgomery ${ }^{1}$ \\ ${ }^{1}$ Naval Postgraduate School, Monterey, CA, USA \\ ${ }^{2}$ Department of Mathematics, Colorado State University, Fort Collins, CO, USA \\ Correspondence to: B. Rutherford (bdruther@nps.edu)
}

Received: 23 March 2011 - Published in Atmos. Chem. Phys. Discuss.: 19 October 2011

Revised: 27 February 2012 - Accepted: 24 May 2012 - Published: 22 June 2012

\begin{abstract}
Recent work has suggested that tropical cyclones intensify via a pathway of rotating deep moist convection in the presence of enhanced fluxes of moisture from the ocean. The rotating deep convective structures possessing enhanced cyclonic vorticity within their cores have been dubbed Vortical Hot Towers (VHTs). In general, the interaction between VHTs and the system-scale vortex, as well as the corresponding evolution of equivalent potential temperature $\left(\theta_{\mathrm{e}}\right)$ that modulates the VHT activity, is a complex problem in moist helical turbulence.

To better understand the structural aspects of the threedimensional intensification process, a Lagrangian perspective is explored that focuses on the coherent structures seen in the flow field associated with VHTs and their vortical remnants, as well as the evolution and localized stirring of $\theta_{\mathrm{e}}$. Recently developed finite-time Lagrangian methods are limited in the three-dimensional turbulence and shear associated with the VHTs. In this paper, new Lagrangian techniques developed for three-dimensional velocity fields are summarized and we apply these techniques to study VHT and $\theta_{\mathrm{e}}$ phenomenology in a high-resolution numerical tropical cyclone simulation. The usefulness of these methods is demonstrated by an analysis of particle trajectories.

We find that VHTs create a locally turbulent mixing environment. However, associated with the VHTs are hyperbolic structures that span between adjacent VHTs or adjacent vortical remnants and represent coherent finite-time transport barriers in the flow field. Although the azimuthally-averaged inflow is responsible for the inward advection of boundary layer $\theta_{\mathrm{e}}$, attracting Lagrangian coherent structures are coincident with pools of high boundary layer $\theta_{\mathrm{e}}$. Extensions of boundary layer coherent structures grow above the boundary layer during episodes of convection and remain with the convective vortices. These hyperbolic structures form initially
\end{abstract}

as boundaries between VHTs. As vorticity aggregates into a ring-like eyewall feature, the Lagrangian boundaries merge into a ring outside of the region of maximal vorticity.

\section{Introduction}

\subsection{The turbulent intensification problem}

\subsubsection{VHT definition and their local dynamics}

Observations show that tropical cyclones are highly asymmetric during their intensification phase. Once mature, only the most intense storms exhibit a strong degree of axial symmetry and even then, only in their inner-core region. Observations show also that intensifying storms are accompanied by bursts of convection, which one may surmise possess significant local buoyancy. When buoyant convection occurs in an environment of non-zero vertical vorticity, updraughts will amplify the vorticity by the process of vortex-tube stretching. There is growing evidence that convective bursts in pre-depression disturbances and tropical depression-strength storms do generate localized cyclonic vorticity anomalies in the lower troposphere whose magnitude exceeds that of the local environment by 1-2 orders of magnitude (Reasor et al., 2005; Sippel et al., 2006; Bell and Montgomery, 2010; Raymond and Lopez-Carrillo, 2010). The rotating deep convective structures that contribute to the stretching of cyclonic vertical vorticity in the low- to mid-troposphere will be hereafter referred to as vortical hot towers (or VHTs) (Hendricks et al., 2004; Dunkerton et al., 2009). Observations and numerical modeling studies suggest that VHTs typically have convective lifetimes on the order of $1 \mathrm{~h}$. Though the lifetime of VHTs is short, the amplified 
vorticity left behind may last much longer and contribute to the aggregation and upscale growth of the emerging cyclonic hurricane.

VHTs are a manifestation of convective instability, generated by moisture fluxes at the air-sea interface, in a rotating environment. Moisture fluxes enhance the moist entropy of the boundary layer above ambient values in the absence of convective- or meso-scale downdrafts, which import low moist entropy from aloft into the boundary layer. The local buoyancy required to support the VHTs is provided by the latent heat of condensation for moist air parcels originating in the atmospheric boundary layer. It is only when a boundary layer parcel is lifted vertically (while conserving its moist entropy) to the so-called "level of free convection," that the parcel will acquire positive buoyancy to accelerate the updraught vertically and stretch vertical vortex tubes. The vertical buoyancy force per unit mass is proportional to the difference between the parcel's virtual temperature and that of its immediate environment. For convenience, the entropy of moist air is often expressed using the variable of equivalent potential temperature $\left(\theta_{\mathrm{e}}\right)$ (Holton, 2004) ${ }^{1}$.

The horizontal length scale of VHTs is on the order of $10 \mathrm{~km}$ and is thought to be associated with a (nonlinear) convective plume scale (Siggia, 1994), in contrast to the most unstable horizontal length scale $(\sim 1 \mathrm{~km}$ or less) that arises in a linearized stability analysis of the moist tropical atmosphere (Lilly, 1960; Emanuel et al., 1994). As discussed in the foregoing paragraph, VHTs feed on heightened $\theta_{\mathrm{e}}$ in the boundary layer. Recent work (Nguyen et al., 2011; Montgomery et al., 2009) indicates that the localized effects of the VHTs include the consumption of convective available potential energy (Holton, 2004) and restoration of convective stability in the local environment. The convective component of a VHT subsides when either (i) the VHT has consumed all of the convective available potential energy in its immediate vicinity, and or (ii) vertical shear differentially advects the columnar structure disrupting the lifecycle. Because of their vortical properties, VHTs contribute to the stirring of $\theta_{\mathrm{e}}$ anomalies and vorticity in the boundary layer through high strain. The precipitation-driven downdrafts that accompany the VHTs in sub-saturated air import low $\theta_{\mathrm{e}}$ from the middle troposphere into the boundary layer. Both of these processes create an environment less favorable for future con-

\footnotetext{
${ }^{1}$ For reference, the connection between the moist entropy $(s)$ and $\theta_{\mathrm{e}}$ is $s=c_{p} \ln \left(\theta_{\mathrm{e}}\right)$, where $c_{p}$ is the specific heat of dry air at constant pressure. For moist-saturated conditions, $\theta_{\mathrm{e}}$ is adequately approximated by the quantity $\theta \exp \left(q L_{v} / c_{p} T\right)$ where $\theta=T\left(p_{0} / p\right)^{\left(R / c_{p}\right)}$ is the "dry" potential temperature, $T$ is thermodynamic temperature, $q$ is the mixing ratio of moist air (expressed in grams of water vapor per kilogram of dry air), $p$ is air pressure, $p_{0}$ is a reference air pressure (1000 mb), $\mathbf{R}$ is the (ideal) gas constant for dry air and $L_{v}$ is the latent heat of condensation. A more precise representation of $\theta_{\mathrm{e}}$ for non-saturated and saturated conditions that includes the virtual temperature effect is given by Bolton (1980) and the Bolton formulation is employed for all $\theta_{\mathrm{e}}$ calculations herein.
}

vective episodes until the boundary layer $\theta_{\mathrm{e}}$ recovers through the air-sea interaction process to a sufficient degree (Nguyen et al., 2011). The spatial distribution of boundary layer $\theta_{\mathrm{e}}$ is therefore an integral component of the VHT dynamics and the spin-up of the system-scale circulation.

\subsubsection{Role of VHTs in the spin up of the mean circulation}

The role of VHTs in the intensification of the larger-scale vortex circulation has been the subject of recent numerical and theoretical investigations, (Hendricks et al., 2004; Montgomery et al., 2006; Nguyen et al., 2008; Shin and Smith, 2008; Nguyen et al., 2011; Montgomery et al., 2010; Fang and Zhang, 2010; Levina and Montgomery, 2010). From a mean-field viewpoint associated with an azimuthal average around the system center of circulation, VHTs have been implicated in two mechanisms for spinning up the mean vortex (Smith et al., 2009; Montgomery and Smith, 2012):

- The first mechanism is associated with the radial convergence of absolute angular momentum above the boundary layer in conjunction with its conservation ${ }^{2}$. The convergence of absolute angular momentum is produced by a system-scale radial gradient of a positive heating rate associated with the VHTs in the presence of enhanced surface moisture fluxes from the underlying ocean ${ }^{3}$. This mechanism has been articulated previously by many authors (e.g., Willoughby, 1979; Schubert and Hack, 1982). It explains why the vortex expands in size (Smith et al., 2009) and may be interpreted in terms of balance dynamics (Bui et al., 2009), wherein the azimuthal mean flow is well approximated by gradient wind and hydrostatic balance ${ }^{4}$.

- The second mechanism is associated with radial convergence of absolute angular momentum within the boundary layer and becomes important in the inner-core

\footnotetext{
${ }^{2}$ The absolute angular momentum, $M=r v+1 / 2 f r^{2}$, is the sum of the planetary angular momentum taken about the storm's rotation axis and the relative angular momentum of the storm's tangential circulation. Here, $r$ denotes radius from the system circulation center, $f$ denotes the Coriolis parameter $(2 \Omega \sin (\phi)$, where $\phi$ is latitude) and $v$ denotes the azimuthally-averaged tangential velocity field, defined relative to the system center.

${ }^{3}$ The heating rate refers to the material derivative of the dry potential temperature, $\theta$, defined in footnote 1 (see also Holton, 2004). Unlike the moist thermodynamic viewpoint discussed in the foregoing subsection in which moist air parcels lifted from the boundary layer rise along moist-adiabats materially conserving their moist entropy (and $\theta_{\mathrm{e}}$ ), in the alternative dry-thermodynamic viewpoint the latent heat that is liberated during the condensation process appears as a forcing term in the thermodynamical equation for $\theta$. These two descriptions are complementary.

${ }^{4}$ See Chapter 3 of Holton (2004) for a definition of gradient and hydrostatic balance.
} 
region of the developing storm. Although absolute angular momentum is not materially conserved in the boundary layer, large wind speeds can be achieved if the radial inflow is sufficiently large to bring the air parcels to small radii with minimal loss of angular momentum. While coupled to the interior flow via the radial pressure gradient at the top of the boundary layer, and still requiring convectively-induced inflow above the boundary layer to increase the radial pressure gradient there, this spin-up pathway is tied to the dynamics of the boundary layer where the flow is not in gradient wind balance over a substantial radial span (Montgomery and Smith, 2012).

\subsubsection{Motivation of this study}

The two-stage model of tropical cyclone intensification proposed by Smith et al. (2009) is thus one that incorporates both the dynamics and thermodynamics of VHTs and their collective effects. In general, the interaction between VHTs and the system-scale vortex, as well as the corresponding evolution of $\theta_{\mathrm{e}}$, is a complex problem in moist helical turbulence ${ }^{5}$ (Levina and Montgomery, 2010). To better understand the structural aspects of the intensification process, a Lagrangian perspective is adopted here for studying the localized stirring around VHTs and their vortical remnants, as well as the evolution and stirring of $\theta_{\mathrm{e}}$. One of the goals of this study is to explore further the system-scale implications of stirring and mixing processes near VHTs and their respective mergers.

Another property of the new model is the stochastic nature in the development and interaction of the VHTs, due to the turbulent nature of the flow. Recalling the rule that the predictability time generally scales with the eddy turnover time of the energy containing eddies, it would seem that meaningful forecasts of the lifecycle of an individual VHT (i.e., formation, growth, merger with neighboring VHTs, and decay) are futile beyond a one hour time scale. Therefore, information about the aggregate contribution of the stochastic turbulent structures would be expected to be obtained by eddy statictics (Weiss and Provenzale, 2008). On the other hand, it is well known that flow boundaries tend to be persistent, even in time-dependent flows, and knowledge of the location of these boundaries within a highly time-dependent flow may improve the localized predictability of the turbulent mixing processes. A second goal of this study is to examine the localized properties of persistent Lagrangian boundaries associated with the VHTs which protect and aggregate regions of vorticity and protect the enhanced $\theta_{\mathrm{e}}$ anomalies that support VHTs.

\footnotetext{
${ }^{5}$ Helicity is the scalar product between the vorticity and velocity vector fields.
}

\subsection{Organization within the turbulence}

Persistent boundaries allow a simplified viewpoint of organized transport, and in steady or weakly time-dependent velocity fields are formed by the stable and unstable manifolds of a trajectory with saddle-type stability, see Ottino (1990); Ide et al. (2002); Malhotra and Wiggins (1999). Flow boundaries for time-dependent flows still share a relationship to the saddle-point geometry for steady flows as they are finite-time manifolds, but the boundaries exist only for finite times, and are limited to finite lengths. Though the flow near VHTs is turbulent, slower processes due to the system-scale convergence are responsible for the flow features outside of VHTs. Consequently, some flow boundaries near VHTs have lifetimes greater than that of the VHT, and provide pathways for stirring of vorticity. Since the boundaries can be visualized in a reference frame moving approximately at the speed of the Lagrangian boundary through the time-dependent flow, these finite-time flow boundaries represent a type of Lagrangian coherent structure (LCS).

\subsubsection{LCS definition}

Lagrangian coherent structures are generally defined as structures which maintain some particular property when advected by the flow. While the Lagrangian reference frame in most meteorological applications follows a coherent feature, the dynamical systems community has recently used the term Lagrangian for following particle trajectories in order to provide a more generalized frame independent view of transport in time-dependent flows (Wiggins, 2005; Haller and Yuan, 2000). Shadden (2006), offers a definition of LCSs commonly accepted in the dynamical systems community as "ridges ${ }^{6}$ of a Lagrangian scalar field." While VHTs are LCSs in the sense of the meteorological definition, their Lagrangian properties in the sense of the latter definition have not been studied extensively. To avoid confusion, the use of the term LCS will refer hereafter to a ridge of a Lagrangian scalar field, and will not be used to refer to a VHT.

Of particular interest for studying mixing are those LCSs which serve as the finite-time analogs of stable and unstable manifolds. Forward time integration yields repelling LCSs while backward time integration yields attracting LCSs, which are the finite-time analogs to stable and unstable manifolds, respectively. Attracting LCSs attract and then stretch a tracer blob, causing stretching, while repelling LCSs split a tracer blob that initially straddles the LCS. The roles of these LCSs in the deformation of tracer blobs are the same as for the invariant manifolds of the steady flow (Ottino, 1990; Haller and Yuan, 2000), and are summarized in Table 1.

\footnotetext{
${ }^{6}$ Ridges of a 2-D scalar field are curves along which the field is locally maximal and the orientation of the curve is everywhere orthogonal to the gradient of the field.
} 
Table 1. Properties of attracting and repelling LCSs.

\begin{tabular}{lccc}
\hline Manifold & LCS & Integration time & Effect on tracers \\
\hline Unstable & Attracting & Backward & Stretching \\
Stable & Repelling & Forward & Splitting \\
\hline
\end{tabular}

\subsubsection{Finite-time Lagrangian methods}

Locating LCSs in 3-D time-dependent flows is challenging, but is aided by a new class of techniques from dynamical systems theory that allow for the detection of LCSs in flows with general time-dependence. Finite-time Lyapunov exponents (FTLE's) were defined by Pierrehumbert (1991) as a method to measure trajectory separation and locate transport barriers, and were later applied to a variety of unsteady fluid flows by Haller and coauthors (Haller and Poje, 1997; Haller and Yuan, 2000; Haller, 2000). The method of FTLE's was shown to be robust under approximation errors of the velocity fields (Haller, 2002). Applications to atmospheric flows include Pierrehumbert (1991); Pierrehumbert and Yang (1993); Ngan and Sheperd (1999); von Hardenberg (2000); Joseph and Legras (2002); Scott et al. (2003); Cohen and Schultz (2005); d'Ovidio (2009); Tang et al. (2010). In Sapsis and Haller (2009), Rutherford et al. (2010b), and Rutherford et al. (2010a), FTLE's have been applied to tropical cyclones.

Though FTLE's easily locate LCSs in time-dependent flows, they do not differentiate between hyperbolicity and shear effectively, and therefore would appear to have limited usefulness in flows with strong shear (d'Ovidio, 2009), such as the inner core region of an intensifying tropical cyclone. A method of separating hyperbolicity from shear in 2-D flows was proposed by Haller and Iacono (2003), and was applied by Rutherford et al. (2010b) to detect LCSs in the presence of large-scale shear. The LCSs found move with the dominant vortex Rossby wave structure, a propogating feature that is not simply the result of advection by the flow, but were shown to be robust across time, and were shown to influence the systematic radial transport of fluid particles within the evolving vortex.

The method of separating shear in two-dimensional flows was extended to 3-D in Rutherford and Dangelmayr (2010). The method was used to compute the Lagrangian boundary separating the eye and eyewall during a mature TC simulation. That study introduced a 3-D flow separation method by decomposing the growth of material elements into several hyperbolic and shear components. Additionally, the hyperbolic fields converged faster than FTLE's and the field associated closely with the horizontal plane eliminated the need for rescaling vertical motions due to the small vertical:horizontal aspect ratio that typifies a tropical cyclone vortex. A specific choice of coordinates adapted to the helical trajectory motion rather than general orthogonal coordinates made the approach of Rutherford and Dangelmayr (2010) easier to im- plement. Whereas the VHT interaction is clearly more complex than the evolution of a single mature hurricane vortex, the coordinate system proposed by Rutherford and Dangelmayr (2010) nonetheless resolves LCSs under shorter timescales. We note that other studies (Truesdell, 1954; Saffman, 1981; Provenzale, 1999; Prieto et al., 2003; Shadden et al., 2006) have investigated the interaction between and entrainment of particles by vortices using different methods, while the ridges of FTLE fields in relation to two vortices was studied by Lapeyre (2002).

In this study, we apply both the methods of Rutherford and Dangelmayr (2010) and FTLEs to a 3-D intensifying tropical cyclone, and examine the LCSs associated with VHTs. We show that vertically coherent hyperbolic LCSs separating the VHTs highlight the presence of flow features which control the organization of VHTs and their vortical remnants, and would thus appear to be important elements of the three-dimensional intensification process. Moreover, the hyperbolic LCSs cannot be isolated by the field of FTLE's even when only considering the two-dimensional flow. The structures we find are long enough to span multiple VHTs, and may contribute to the upscale organization proposed by Montgomery et al. (2006), Nguyen et al. (2008), and Shin and Smith (2008).

\subsubsection{Outline}

The outline of the remainder of this paper is as follows. Section 2 provides an overview of the coordinate system and the Lagrangian methods used in this 3-D study. In Sect. 3, the meteorological model from which the velocity data are calculated is described, along with numerical details regarding trajectory calculations. In Sect. 4, we describe the structure of the flow field in the vicinity of VHTs through LCSs and apply a trajectory analysis. In Sect. 5, we examine the timeevolution of the LCSs in and above the boundary layer. We conclude, in Sect. 6, with further remarks on the relation between Lagrangian coherent structures and VHTs, and provide an outlook on future studies stimulated by the results of this paper.

\section{Mathematical methods}

This section summarizes the mathematical tools employed to explore the Lagrangian perspective of intensification. We denote all vector quantities using boldface, and all matrices in capitalized boldface font. An asterik refers to a transposed vector or matrix.

The Lagrangian trajectory based approaches in previous studies (Haller and Yuan, 2000; Haller, 2002; Shadden et al., 2005; Rutherford and Dangelmayr, 2010) utilize particle trajectories $\boldsymbol{x}(t)$, which evolve according to the flow map after time $T$ (Ottino, 1990),

$\boldsymbol{x}\left(t_{0}\right) \rightarrow \boldsymbol{x}\left(t_{0}, t_{0}+T\right)$. 
The methods detect LCSs by measuring Lagrangian stretching along trajectories through the linearized growth of perturbations relative to an initial particle position. The perturbation vector $\xi$ evolves according to the variational equation

$\dot{\boldsymbol{\xi}}=\nabla \boldsymbol{u}(\boldsymbol{x}(t), t) \boldsymbol{\xi}$,

where $\boldsymbol{u}(\boldsymbol{x}, t)$ is the fluid velocity of $\boldsymbol{x}(t)$ at time $t$. Finitetime Lyapunov exponents (FTLE's) and similar metrics measure the linearized growth as the separation of nearby trajectories, and provide scalar fields which show LCSs corresponding to stable and unstable manifolds as ridges. Computing the FTLE forward $(T>0)$ and backward $(T<0)$ in time allows detection of forward time repelling and attracting material lines, respectively.

While FTLE's are an efficient measure of trajectory separation, they do not differentiate between hyperbolicity and shear (d'Ovidio, 2009). The study of LCSs in sheared flow for a 2-D nondivergent barotropic vortex (Rutherford et al., 2010b) showed that FTLE's were not well suited for rotational flows when the system-scale radial derivative of angular velocity $\partial \omega / \partial r$ was large. For the experiment used in this study, the shear is small enough so that FTLE-fields show well defined ridges in many cases. In regions with strong horizontal shear, the orthogonal growth rate of small perturbations caused by persistent strain can still be isolated by solutions of Eq. (2) as discussed in the following.

We now consider the case where $\boldsymbol{u}(\boldsymbol{x}, t)$ is a 3-D timedependent velocity field. The Lagrangian velocity direction is given by the unit tangent vector

$\boldsymbol{t}=\frac{\boldsymbol{u}}{|\boldsymbol{u}|}$,

evaluated along trajectories. A moving frame of reference for Eq. (2) is introduced along a trajectory by setting

$\xi=\mathbf{T}(\boldsymbol{x}(t), t) \boldsymbol{\eta}$,

where the columns of $\mathbf{T}$ are the orthogonal unit vectors $\boldsymbol{t}, \boldsymbol{n}, \boldsymbol{b}$,

$\mathbf{T}(\boldsymbol{x}, t)=[\boldsymbol{t}(\boldsymbol{x}, t), \boldsymbol{n}(\boldsymbol{x}, t), \boldsymbol{b}(\boldsymbol{x}, t)]$.

Here $\boldsymbol{b}=\boldsymbol{t} \times \boldsymbol{n}$ is the binormal vector, and $\boldsymbol{n}$, referred to as normal vector, is any vector in the normal plane that varies smoothly with $(\boldsymbol{x}, t)$. We refer to the coordinate frame with $\boldsymbol{t}$ defined by Eq. (3) and $\boldsymbol{n}$ and $\boldsymbol{b}$ chosen such that the columns of $\mathbf{T}$ are orthonormal as the tangent-normal-binormal (TNB) coordinate frame. A possible choice for $\boldsymbol{n}$ is the direction of the principal normal of the trajectory but in this study we choose a direction which is rotated to the helical particle motion and simplifies the numerical implementation. The transformed system for $\boldsymbol{\eta}$ has the form

$\dot{\boldsymbol{\eta}}=[\mathbf{A}(\boldsymbol{x}(t), t)+\mathbf{B}(\boldsymbol{x}(t), t)] \boldsymbol{\eta}$, where

$\mathbf{A}(\boldsymbol{x}, t)=\mathbf{T}^{*}(\nabla \boldsymbol{u}) \mathbf{T}-\mathbf{T}^{*}\left(\mathbf{T}_{x} \dot{\boldsymbol{x}}\right)$

with

$\mathbf{T}_{x} \dot{\boldsymbol{x}}=\left[(\nabla \boldsymbol{u}) \boldsymbol{t}-\left[\boldsymbol{t}^{*}(\nabla \boldsymbol{u}) \boldsymbol{t}\right] \boldsymbol{t},(\nabla \boldsymbol{n}) \boldsymbol{u},(\nabla \boldsymbol{b}) \boldsymbol{u}\right]$,

and $\quad \mathbf{B}(\boldsymbol{x}, t)=-\left[\boldsymbol{b}_{1}, \mathbf{T}^{*} \boldsymbol{n}_{t}, \mathbf{T}^{*} \boldsymbol{b}_{t}\right] \quad$ with $\quad \boldsymbol{b}_{1}=$ $(1 /|\boldsymbol{u}|)\left[0, \boldsymbol{n}^{*} \boldsymbol{u}_{t}, \boldsymbol{b}^{*} \boldsymbol{u}_{t}\right]^{*}$. $\mathbf{B}(\boldsymbol{x}, t)$ contains all terms of the transformed matrix that depend on the explicit time derivatives of $\boldsymbol{u}, \boldsymbol{n}, \boldsymbol{b}$ (indicated by the subscript $t$ ). The time derivatives along trajectories, which would be present in a steady flow, are given by Eq. (8) and are incorporated into $\mathbf{A}(\boldsymbol{x}, t)$. In the case of steady velocity fields, $\mathbf{B}$ vanishes and Eq. (6) reduces to

$\dot{\eta}=\mathbf{A}(\boldsymbol{x}(t), t) \boldsymbol{\eta}$.

As in previous studies (Rutherford and Dangelmayr, 2010; Rutherford et al., 2010b), the time-variation of fluid velocities along trajectories is small compared to the spatial derivatives, which are included in the terms of $\mathbf{A}$ for short integration times. Thus, we use Eq. (9) to approximate finite-time solutions of the transformed variational system. Combining the two terms of which $\mathbf{A}$ is composed in Eq. (7) yields $\mathbf{A}=\left[\boldsymbol{a}_{1}, \mathbf{T}^{*} \boldsymbol{a}_{2}, \mathbf{T}^{*} \boldsymbol{a}_{3}\right]$, where

$\boldsymbol{a}_{1}=\left[\boldsymbol{t}^{*}(\nabla \boldsymbol{u}) \boldsymbol{t}, 0,0\right]^{*}$,

$\boldsymbol{a}_{2}=(\nabla \boldsymbol{u}) \boldsymbol{n}-(\nabla \boldsymbol{n}) \boldsymbol{u}$,

$\boldsymbol{a}_{3}=(\nabla \boldsymbol{u}) \boldsymbol{b}-(\nabla \boldsymbol{b}) \boldsymbol{u}$.

\subsection{Transformation to upper triangular form and solu- tion of the variational system}

In the 2-D setting (Haller and Iacono, 2003), the matrix $\mathbf{A}$ is upper triangular, and $A_{22}$ is the orthogonal strain rate. The component of solution corresponding to hyperbolic separation is found by directly integrating $A_{22}$. Outside of vortex cores and regions of high shear, the hyperbolic separation coincides with FTLE's. The hyperbolic solution only incorporates orthogonal separation and neglects shear. Results from a 2-D barotropic turbulence simulation (Haller and Iacono, 2003) show the role of the hyperbolic component as a transport barrier between vortices.

In contrast to the 2-D case of Haller and Iacono (2003) and Rutherford et al. (2010b), the matrix A is not upper triangular, and thus Eq. (9) is not solvable unless the flow is integrable. Still, the transformed variational equation reduces to a 2-D system on the normal plane, $\eta^{\perp}=\left(\eta_{2}, \eta_{3}\right)^{*}$, which satisfies $\dot{\eta}^{\perp}=\mathbf{A}^{\perp} \boldsymbol{\eta}$ with

$\mathbf{A}^{\perp}=\left(\begin{array}{ll}A_{22} & A_{23} \\ A_{32} & A_{33}\end{array}\right)$.

The normal plane system is solvable if there is a continuous transformation orienting the normal plane so that $\mathbf{A}^{\perp}$ is upper 
triangular. To obtain upper triangular form we apply a timedependent orthogonal transformation in the normal plane.

Let $\phi(t)$ be a solution to the differential equation

$\dot{\phi}=\frac{1}{2}\left(A_{33}-A_{22}\right) \sin 2 \phi+A_{23} \sin ^{2} \phi-A_{32} \cos ^{2} \phi$,

and $\mathbf{R}(\phi)$ the rotation matrix

$\mathbf{R}(\phi)=\left(\begin{array}{r}\cos \phi \sin \phi \\ -\sin \phi \cos \phi\end{array}\right)$.

The transformation $\eta^{\perp}=\mathbf{R}(\phi(t)) \widetilde{\eta}^{\perp}$ transforms the normal plane system to

$\dot{\tilde{\eta}}^{\perp}=\widetilde{\mathbf{A}}^{\perp} \widetilde{\boldsymbol{\eta}}^{\perp}$,

where $\widetilde{\mathbf{A}}^{\perp}=\mathbf{R}(\phi) \cdot \mathbf{A}^{\perp}$ is upper triangular. Thus, dropping the tilde, we may assume that $\mathbf{A}$ in Eq. (9) has the form

$\mathbf{A}(\boldsymbol{x}(t), t)=\left(\begin{array}{ccc}A_{11} & A_{12} & A_{13} \\ 0 & A_{22} & A_{23} \\ 0 & 0 & A_{33}\end{array}\right)$,

and the transformed variational system can be solved by direct integration. The fundamental matrix whose columns are linearly independent solutions for the system (9) with $\mathbf{A}$ given by Eq. (14) is found by direct integration as

$\boldsymbol{\Psi}\left(t, t_{0}\right)=\left(\begin{array}{ccc}\Psi_{11}\left(t, t_{0}\right) & \Psi_{12}\left(t, t_{0}\right) & \Psi_{13}\left(t, t_{0}\right) \\ 0 & \Psi_{22}\left(t, t_{0}\right) & \Psi_{23}\left(t, t_{0}\right) \\ 0 & 0 & \Psi_{33}\left(t, t_{0}\right)\end{array}\right)$,

where the diagonal elements can be written as

$\Psi_{i i}\left(t, t_{0}\right)=\exp \left(\int_{t_{0}}^{t} A_{i i}(\tau) d \tau\right)$

and the off-diagonal elements as

$$
\begin{aligned}
\Psi_{12}= & \int_{t_{0}}^{t} \exp \left(\int_{s}^{t} A_{11}(\tau) d \tau\right) \\
& \exp \left(\int_{t_{0}}^{s} A_{22}(\tau) d \tau\right) A_{12}(s) d s, \\
\Psi_{23}= & \int_{t_{0}}^{t} \exp \left(\int_{s}^{t} A_{22}(\tau) d \tau\right) \\
& \exp \left(\int_{t_{0}}^{s} A_{33}(\tau) d \tau\right) A_{23}(s) d s, \\
\Psi_{13}= & \int_{t_{0}}^{t} \exp \left(\int_{s}^{t} A_{11}(\tau) \tau\right)\left[\Psi_{23}\left(s, t_{0}\right) A_{12}(s)\right. \\
& \left.\quad+\exp \left(\int_{t_{0}}^{s} A_{33}(\tau) d \tau\right) A_{13}(s)\right] d s .
\end{aligned}
$$

\section{Horizontally aligned normal vector}

For a 3-D hurricane flow, the particle motion in the eyewall can be described qualitatively as helical, with rotational and vertical components. For this reason, we choose a TNBsystem that is related to this helical motion and simplies the numerical implementation. The appropriate choice for $\boldsymbol{n}$ is the outward normal vector

$\boldsymbol{n}=\boldsymbol{u}_{\mathrm{h}}^{\perp} /\left|\boldsymbol{u}_{\mathrm{h}}\right|$,

where $\boldsymbol{u}_{\mathrm{h}}=(u, v, 0)^{*}$ is the horizontal component of the velocity, and $\boldsymbol{u}_{\mathrm{h}}^{\perp}=(-v, u, 0)^{*}$. In this case the binormal is given by

$\boldsymbol{b}=\frac{1}{|\boldsymbol{u}|\left|\boldsymbol{u}_{\mathrm{h}}\right|}\left(-u w,-v w,\left|\boldsymbol{u}_{\mathrm{h}}\right|^{2}\right)^{*}$,

and the entries $A_{i j}$ with $i>1$ in Eq. (14) become

$$
\begin{aligned}
A_{12}= & \frac{1}{|\boldsymbol{u}|\left|\boldsymbol{u}_{\mathrm{h}}\right|}\left\{\left(u^{2}-v^{2}\right)\left(u_{y}+v_{x}\right)+2 u v\left(v_{y}-u_{x}\right)\right. \\
& \left.\quad+u w\left(v_{z}+w_{y}\right)-v w\left(u_{z}+w_{x}\right)\right\}, \\
A_{13}= & \frac{1}{|\boldsymbol{u}|^{2}\left|\boldsymbol{u}_{\mathrm{h}}\right|}\left\{( | \boldsymbol { u } _ { \mathrm { h } } | ^ { 2 } - w ^ { 2 } ) \left(v\left(v_{z}+w_{y}\right)\right.\right. \\
& \left.+u\left(u_{z}+w_{x}\right)\right)-2 w\left(u^{2} u_{x}+v^{2} v_{y}\right) \\
& \left.-2 u v w\left(u_{y}+v_{x}\right)\right\}, \\
A_{22}= & \frac{1}{\left|\boldsymbol{u}_{\mathrm{h}}\right|^{2}}\left\{u^{2} v_{y}+v^{2} u_{x}-u v\left(u_{y}+v_{x}\right)\right\}, \\
A_{23}= & \frac{|\boldsymbol{u}|\left(u v_{z}-v u_{z}\right)}{\left|\boldsymbol{u}_{\mathrm{h}}\right|^{2}}, \\
A_{32}= & \frac{1}{|\boldsymbol{u}|\left|\boldsymbol{u}_{\mathrm{h}}\right|^{2}}\left\{2 w \left(v^{2} v_{x}-u^{2} u_{y}+\right.\right. \\
& \left.u v\left(u_{x}-v_{y}\right)\right)+w^{2}\left(v u_{z}-u v_{z}\right) \\
& \left.+\left|\boldsymbol{u}_{\mathrm{h}}\right|^{2}\left(u w_{y}-v w_{x}+w\left(u_{y}-v_{x}\right)\right)\right\}, \\
A_{33}= & \frac{1}{|\boldsymbol{u}|^{2}\left|\boldsymbol{u}_{\mathrm{h}}\right|^{2}}\left\{w^{2}\left(u^{2} u_{x}+v^{2} v_{y}+u v\left(u_{y}+v_{x}\right)\right)\right. \\
& -\left|\boldsymbol{u}_{\mathrm{h}}\right|^{2} w\left(u\left(u_{z}+w_{x}\right)+v\left(v_{z}+w_{y}\right)\right) \\
& \left.+\left|\boldsymbol{u}_{\mathrm{h}}\right|^{4} w_{z}\right\} .
\end{aligned}
$$

This particular choice of $\boldsymbol{n}$ orients the coordinate system so that $\boldsymbol{n}$ lies initially in the $\mathrm{x}-\mathrm{y}$ plane with the initial condition $\mathbf{R}(0)=\mathbf{I}$. Since the normal plane is allowed rotation after the initial time, the $\Psi$ fields which depend on the normal plane account for 3-D separation, with $\Psi_{22}$ measuring approximately the outward component of the strain. By using the continuous coordinate transformation $\phi$, the orientation of the $\eta$ coordinates is preserved along trajectories. We note that the basis used to define the components of $\Psi$ is not Galilean invariant and may require a storm-relative coordinate to be suitable for a translating vortex. However, $\Psi_{22}$ maintains its orientation along trajectories and does not depend on the direction of $\boldsymbol{n}$. Rutherford and Dangelmayr (2010) found that the transformation $\mathbf{R}(\phi)$ in Eq. (12) was continuous for trajectories in a simulated mature tropical cyclone. For this study, we consider $\boldsymbol{\Psi}$ to be a useful diagnostic 
primarily outside VHTs, and we use $\Psi_{22}$ to isolate LCSs that reside between VHTs. For further mathematical details we refer to Rutherford and Dangelmayr (2010).

\section{Model description and numerical implementation of Lagrangian fields}

\subsection{Setup}

The model used to generate data for this study is the fifth generation Penn State/NCAR mesoscale model (MM5), Grell et al. (1995) and Dudhia (1993). The model run is a fully 3-D nonhydrostatic simulation of an idealized tropical cyclone on an f-plane. The model employs a bulk aerodynamic scheme for representing the fluxes of sensible heat, moisture, and momentum between the atmosphere and the ocean. The wind-speed dependence of the heat and moisture fluxes from the underlying ocean surface is retained in this experiment. The model calculations were initialized using a convectively neutral environment and an axisymmetric warm-core, cloudfree vortex with a maximum surface tangential velocity of $15 \mathrm{~m} \mathrm{~s}^{-1}$ at a radius of $135 \mathrm{~km}$ (Nguyen et al., 2008). The intensification of the vortex is highlighted in Fig. 1 by plotting the local maximum of horizontal wind speed as a function of time. The model data used in this study is a higher resolution version of experiment 12 of Montgomery et al. (2009).

The model run used in this study utilized four nested grids, and 3-D velocity data is taken only on the innermost $300 \mathrm{~km}$ square grid with $\mathrm{x}-\mathrm{y}$ grid spacing of $1.67 \mathrm{~km}$. Vertical coordinates are given on $\sigma$-levels, and vary in time, with an output time-step of $2 \mathrm{~min}$. Trajectory integrations are performed using a fourth order Runge-Kutta scheme using the full 4-D velocity fields, on grids of evenly spaced points in a box of size $220 \mathrm{~km}$ by $220 \mathrm{~km}$ by $16 \mathrm{~km}$ in $x, y$, and $z$ respectively, with horizontal grid-spacing of $1 \mathrm{~km}$, and vertical grid-spacing of $250 \mathrm{~m}$.

\subsection{Simulation summary}

Though the initial condition is axisymmetric, asymmetries develop quickly around $8 \mathrm{~h}$ in the form of near-columnar convective structures posessing strong cyclonic vorticity in their cores. The asymmetric stage begins at $\sim 8 \mathrm{~h}$ with modestly high $\theta_{\mathrm{e}}$ in an $80 \mathrm{~km}$ ring (Fig. 2c), which, in conjunction with the frictional convergence in the boundary layer, induces convective instability and the formation of rotating deep convection beginning just before $10 \mathrm{~h}$ coincident with the first sharp rise in intensity (Fig. 1).

As discussed in the Introduction, these convective structures are called VHTs, and are identified as localized regions of enhanced vorticity. In this simulation, twelve VHTs appear prior to rapid intensification. As the VHTs interact they comprise the bulk of the system scale radial gradient of latent heating. This heating gradient leads to a radial influx of vertical vorticity (Bui et al., 2009), and $\theta_{\mathrm{e}}$ acquired through

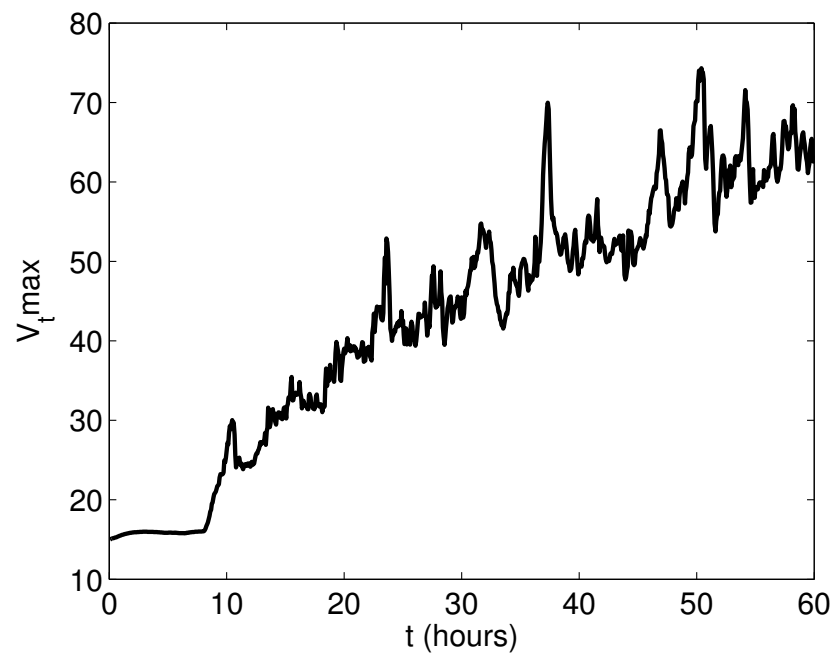

Fig. 1. Intensification of the simulated vortex is shown by the local maximum of horizontal wind speed $V_{t, \max }$ as a function of time from $t=0$ to $t=60 \mathrm{~h}$.

the air-sea interaction process. An eye-like feature forms at approximately $40 \mathrm{~h}$, Fig. 2e, with a ring of maximal cyclonic vorticity evident near $40 \mathrm{~km}$ radius and the highest $\theta_{\mathrm{e}}$ located interior to this radius at approximately $30-35 \mathrm{~km}$.

As the vortex intensifies, the number of VHTs progressively decreases as the vortex attains maturity. The maximal horizontal wind speed increases from $15 \mathrm{~m} \mathrm{~s}^{-1}$ at $5 \mathrm{~h}$ to over $60 \mathrm{~m} \mathrm{~s}^{-1}$ at $50 \mathrm{~h}$. By the end of the rapid intensification period (approximately $60 \mathrm{~h}$ and onwards, not shown), no more than three VHTs are active around the circulation centre. In the mature phase, the evolution of the vortex core is characterized by an approximately axisymmetric circulation superimposed on which are small, but finite-amplitude vortex Rossby waves that propagate azimuthally, radially, and vertically on the mean potential vorticity gradient of the systemscale vortex coupled to the to the boundary layer and convection (Wang, 2002; Chen and Yau, 2003; Chen et al., 2003; Smith et al., 2008). In this study we examine fields from times of $t=0$ to $50 \mathrm{~h}$ during the main intensification phase.

\subsection{Lagrangian field computations}

Both FTLE's and $\boldsymbol{\Psi}$ fields are computed from grids of trajectories. Due to the small vertical to horizontal aspect ratio of the model output domain, the inclusion of the vertical component in the computation of FTLE's reduces the resolution of ridges under a fixed integration time. An alternate "planar" FTLE can be computed from separation of trajectories projected onto a plane. For the "planar" FTLE, we compute the standard 2-D FTLE on the alternate flow map derived from the standard flow map in Eq. (1). This flow map can be 


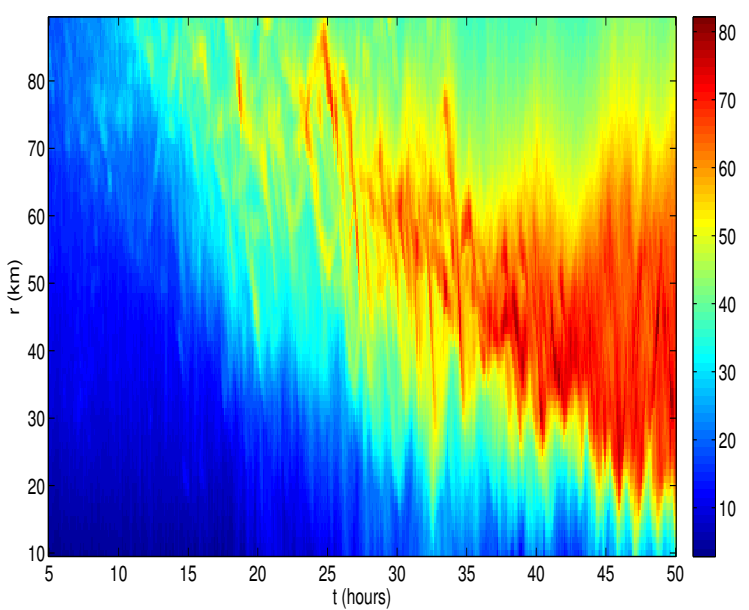

(a) $V_{t, \max }(\mathrm{m} / \mathrm{s})$

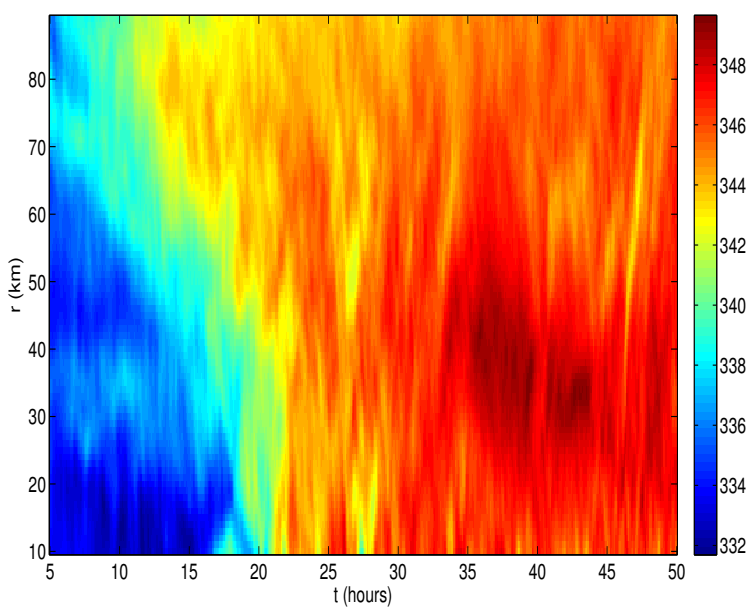

(c) $\theta_{e}(\mathbf{K})$

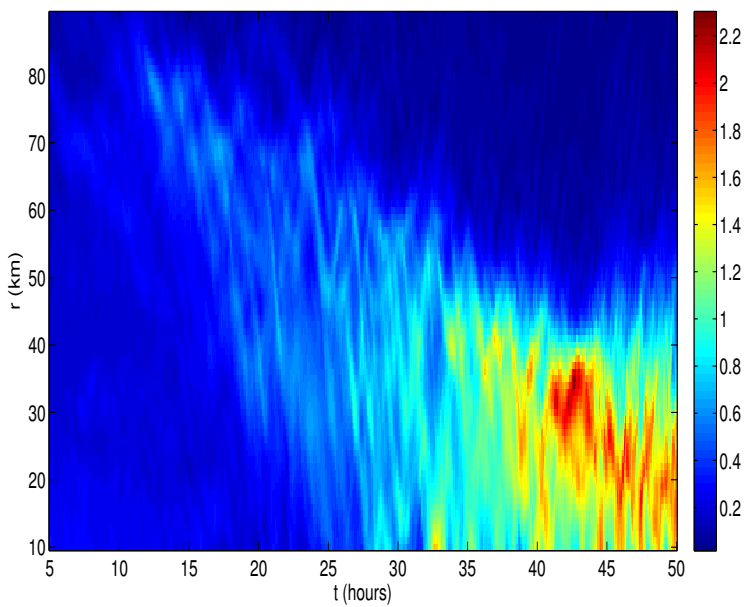

(e) $\zeta\left(s^{-1}\right)$

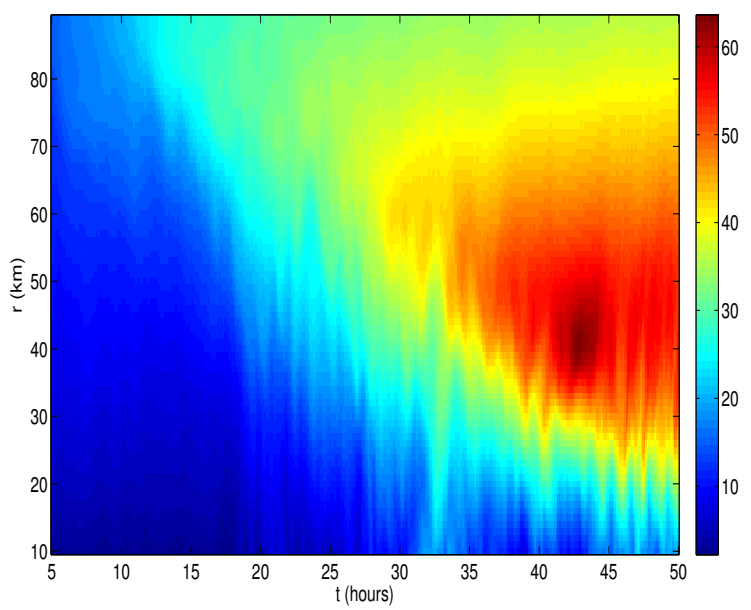

(b) $V_{t, \text { mean }}(\mathrm{m} / \mathrm{s})$

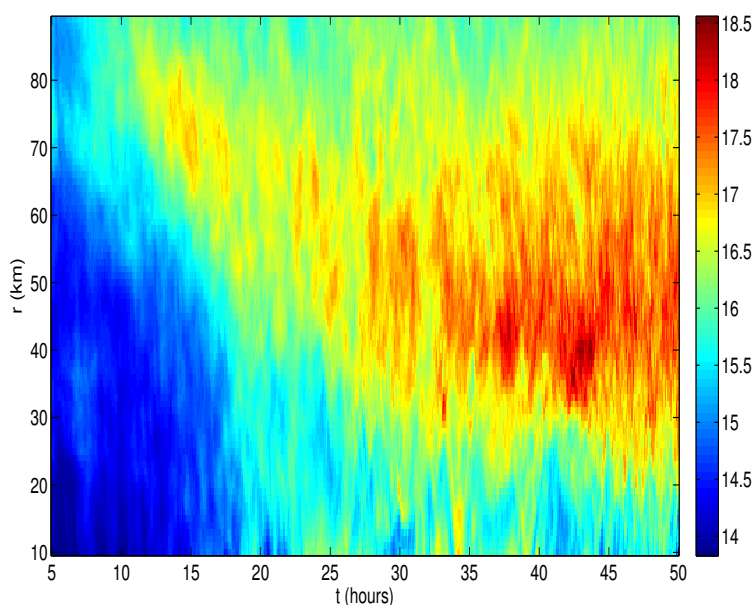

(d) FTLE's

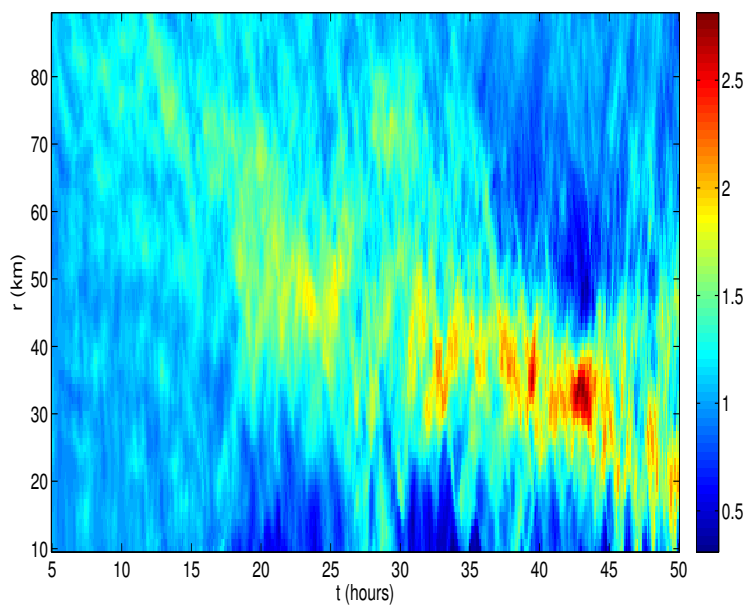

(f) $\Psi_{22}$

Fig. 2. Hovmoller plots (radial profile versus time) of azimuthally averaged $V_{\tan , \max }(\mathbf{a}), V_{\mathrm{tan}, \mathrm{mean}}$ (b), $\theta_{\mathrm{e}}$ (c), FTLE's (d), $\zeta$ (e), and $\Psi_{22}$ (f). The $V_{\tan \text {,mean }}$, FTLE, $\Psi_{22}, \theta_{\mathrm{e}}$, and $\zeta$ values are averaged over the $z=40 \mathrm{~m}$ horizontal plane, while $V_{\tan \text {, max }}$ is a maximum over all heights and azimuths at each radius. 


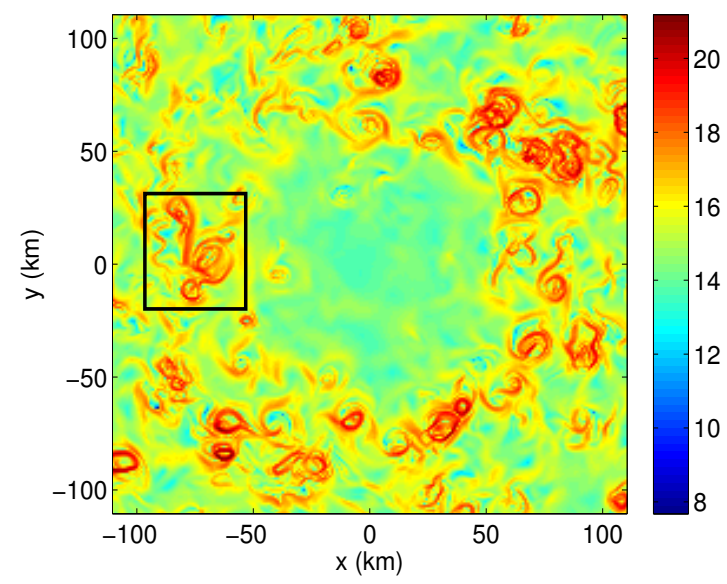

(a)

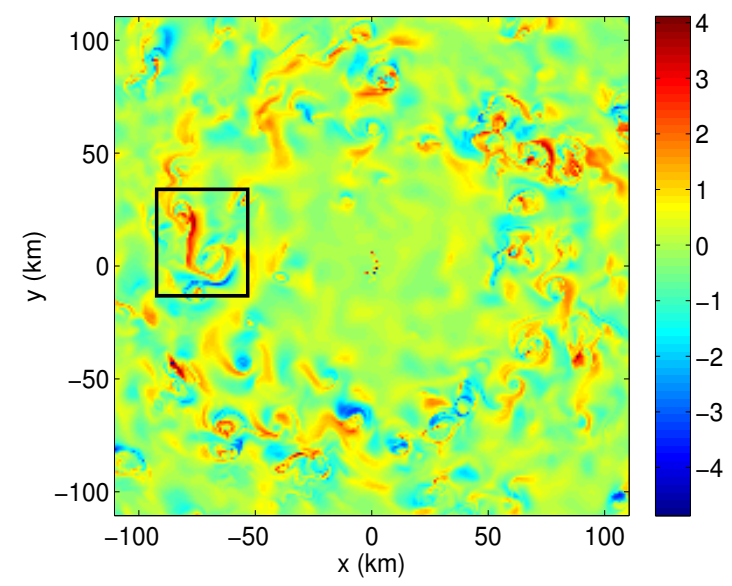

(c)

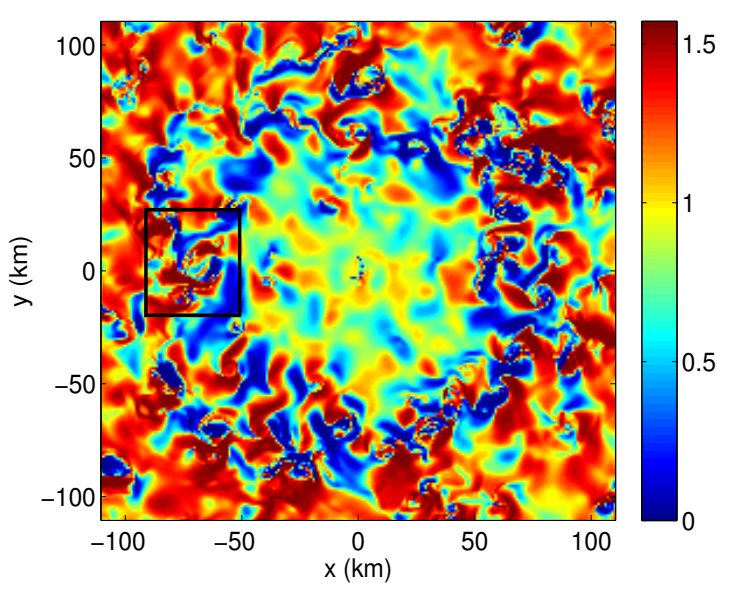

(e)

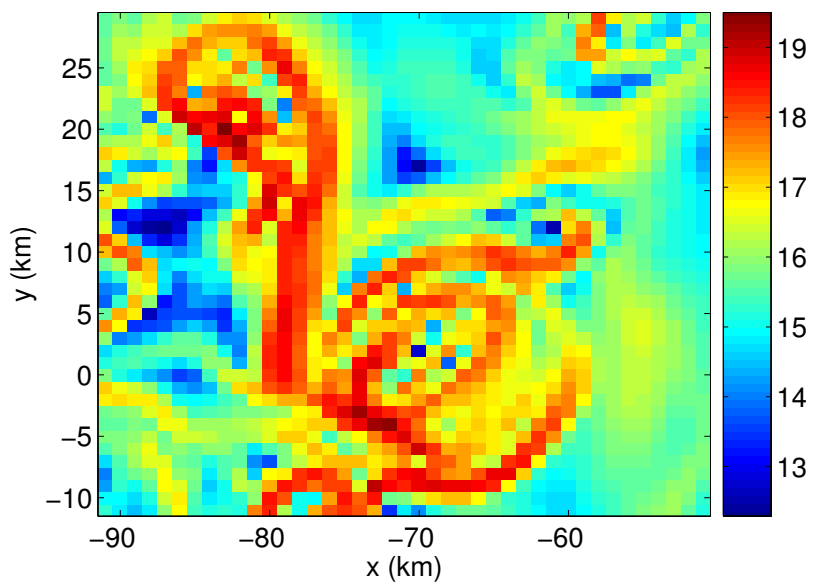

(b)

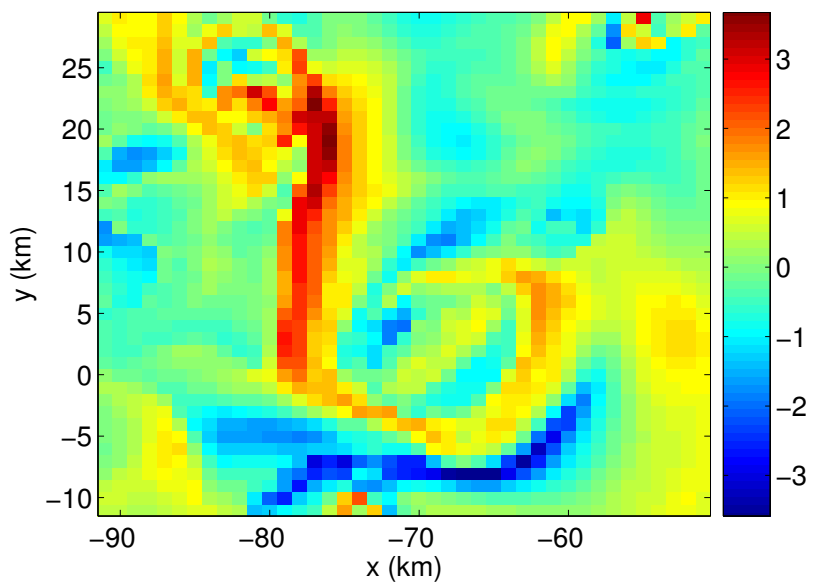

(d)

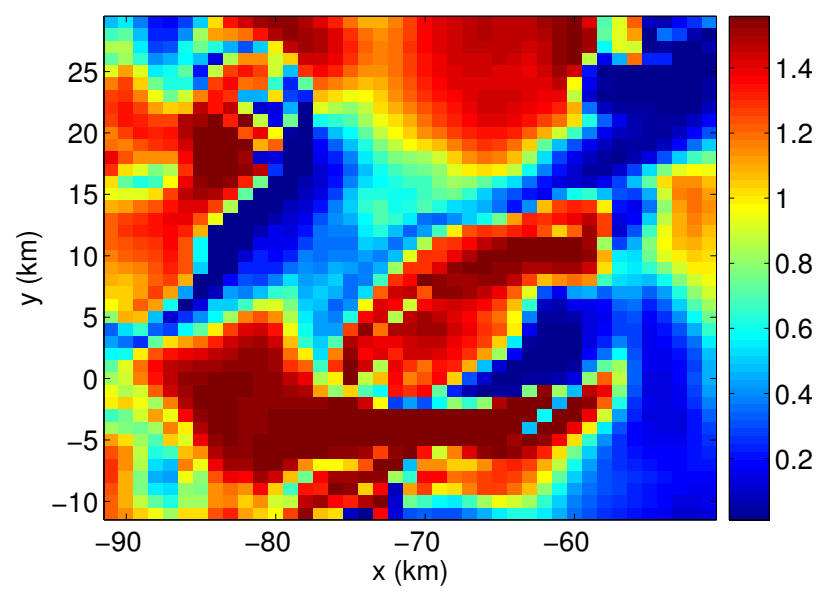

(f)

Fig. 3. Planar FTLE, $\Psi_{22}$, and $\varphi$ fields in (a), (c), and (e), respectively, at initial time of $10 \mathrm{~h}$ with integration time of $1 \mathrm{~h}$ at $1 \mathrm{~km}$ height. A zoom into a particular structure from the boxes in (a), (c), and (e) are shown in (b), (d), and (f).

defined by

$\boldsymbol{x}\left(t_{0}, t_{0}+T\right) \rightarrow \boldsymbol{x}^{\prime}\left(t_{0}, t_{0}+T\right)=\left(\begin{array}{c}x\left(t_{0}, t_{0}+T\right) \\ y\left(t_{0}, t_{0}+T\right) \\ c\end{array}\right)$ where $c$ is constant. The "planar" FTLE resolves ridges much faster than the 3-D FTLE field. In contrast, the $\boldsymbol{\Psi}$ fields are computed using the full 3-D trajectories, and the horizontal 
separation is defined by the construction of $\Psi_{22}$, and does not require any projection of trajectories onto a horizontal plane.

Horizontal shear is computed as in Rutherford and Dangelmayr (2010) by the angle of rotation $\varphi$ of the $\eta_{2}$ variable onto the $\eta_{1}$ variable subspace defined by $\varphi=\arctan \left(\Psi_{12} / \Psi_{22}\right)$. Maximal shear is defined by a complete rotation of the $\eta_{2}$ subspace onto the $\eta_{1}$ subspace, which is indicated by values of $\varphi$ near $\pi / 2$. Lagrangian vortices show little rotation of the $\eta_{2}$ subspace onto the $\eta_{1}$ subspace, and are therefore shown by $\varphi \approx 0$.

LCSs can be seen as maximal ridges of fixed-time Lagrangian scalar fields, and can be tracked over varying initial times. Attracting LCSs are found mainly along the boundaries of regions with high $\theta_{\mathrm{e}}$. VHTs travel toward the center of the repelling LCSs, and the planar vorticity pools associated with the VHT tend not to be stretched horizontally.

For the Lagrangian field computations, the initial time, $t_{0}$, is varied between 5 and $50 \mathrm{~h}$, which captures the model time interval in which VHTs organize vorticity into a symmetric ring-like structure. The convention for Lagrangian fields is to define the Lagrangian field at the initial time of integration, $t$, since they are integrated quantities and incorporate velocities from the interval $(t, t+T)$. The Lagrangian fields at time $t$ are compared to Eulerian fields at a fixed time $t$, so $t$ refers to both initial time of the Lagrangian fields and model output time of Eulerian fields.

To relate the LCSs to vorticity and $\theta_{\mathrm{e}}$ in both forward and backward time, the ridges of the Lagrangian fields are approximated by contours and are overlaid along with vorticity contours on $\theta_{\mathrm{e}}$ fields. We show LCSs which have Lagrangian values that exceed a threshold of over $80 \%$ of the maximum Lagrangian value at that initial time.

\section{Structure of the flow field}

\subsection{Fixed-time Lagrangian fields}

Though Lagrangian values are defined along any trajectory, and fields are computed on 3-D grids, we show the Lagrangian fields on z-levels for ease of visualization of the LCSs. To illustrate the spatial forms of these structures, we show in Fig. 3 the planar FTLE, $\Psi_{22}$, and $\varphi$ fields at $z=1 \mathrm{~km}$ and $10 \mathrm{~h}$, along with zooms highlighting a particular structure to compare the Lagrangian fields. The FTLE maxima occur at any point of high separation, including shear lines surrounding vortices and hyperbolic lines, while the $\Psi_{22}$ field isolates hyperbolic separation. We note that $\Psi_{22}$ ridges are generally in regions of low shear between VHTs and do not enclose VHTs. We specifically refer to these LCSs as $\boldsymbol{\Psi}$ LCSs to distinguish them from LCSs that are associated with FTLE's. The particular structure highlighted in the closeup views shows that the FTLE ridge encloses two rings of vorticity, in addition to where it coincides with the $\boldsymbol{\Psi}$-LCS. The enclosures around the VHTs are in regions of high horizon- tal shear, so the FTLE ridge surrounding them does not have hyperbolic stability.

\subsection{Sensitivity to integration time}

The relationship between the time and length scales of VHTs and those of the LCSs near them drive our choice of integration time for the LCSs. A longer integration time resolves and lengthens LCSs. However, spurious structures and excessive filamentation may emerge for long integration times due to the time-dependence. A short integration time will not resolve as many LCSs, but will not show spurious structures and requires less computation time.

The sensitivity of Lagrangian fields to integration time can be seen in Fig. 4, which shows the planar FTLE field for integration times of $30 \mathrm{~min}, 1 \mathrm{~h}, 2 \mathrm{~h}$, and $4 \mathrm{~h}$, respectively. The longer integration times resolve LCSs better, but introduce a far more complicated structure. However, even under a $4 \mathrm{~h}$ integration time, there are some LCSs which are isolated, particularly in the interior of the circulation. The existence of isolated LCSs suggests that some flow characteristics are coherent through the entire integration time.

The lifespan of most LCSs based on visual inspection is approximately the same as the $1 \mathrm{~h}$ lifetime of VHTs. The merger or disappearance of VHTs, as seen by the end of intense convection and decay of maximum vorticity at lower levels, is coincident with a change in the flow field which may alter the saddle-type geometry of the LCS and cause them to merge or split. However, the flow field defined by a single VHT does not neccessarily determine the flow field in relation to a saddle between the vortices. Since LCSs reside outside VHTs, the death or merger of VHTs does not imply the disappearance or merger of their associated LCSs, allowing some LCSs to persist for much longer than $1 \mathrm{~h}$. Due to the complexity of the flow, the influences of convection, planar dispersion, and differential rotation associated with the horizontal swirling flow complicate the LCS structure as integration time is increased (Rutherford et al., 2010b). Thus the LCSs visualized under short integration times do not necessarily act as flow boundaries for longer integration times. For this reason, we primarily consider LCSs which are resolved under short integration times, yet persist longer than the typical VHT.

Since the representative lifetime of a VHT is $1 \mathrm{~h}$ and LCSs remain isolated for this integration time, we have chosen $T=1 \mathrm{~h}$ as the primary integration time to show the timeevolution of LCSs in Sect. 5. This choice limits mergers or splitting of the LCSs as the VHTs die, yet is still sufficiently long to reveal LCSs that span between the VHTs, including LCSs which are associated with vortical remnants and have a lifespan on the order of $10 \mathrm{~h}$. Our approach to understand time-dependent stirring is to consider LCSs which are well resolved under a short integration time and coherent across varying initial times. 


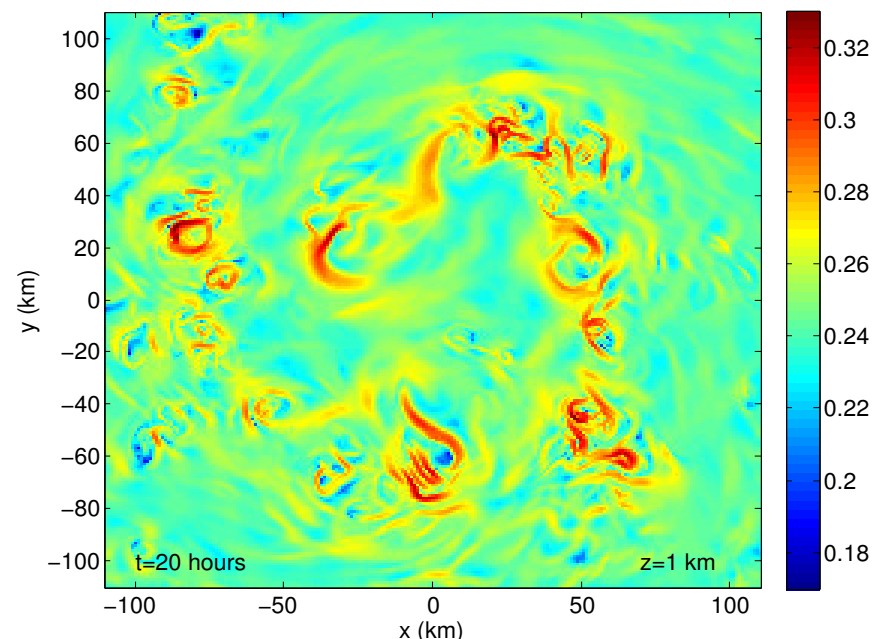

(a)

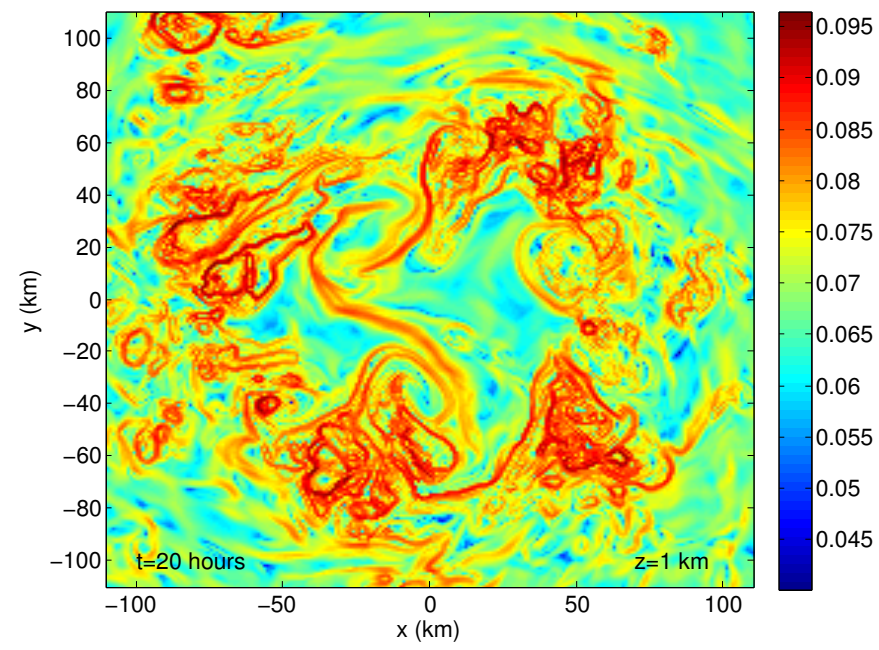

(c)

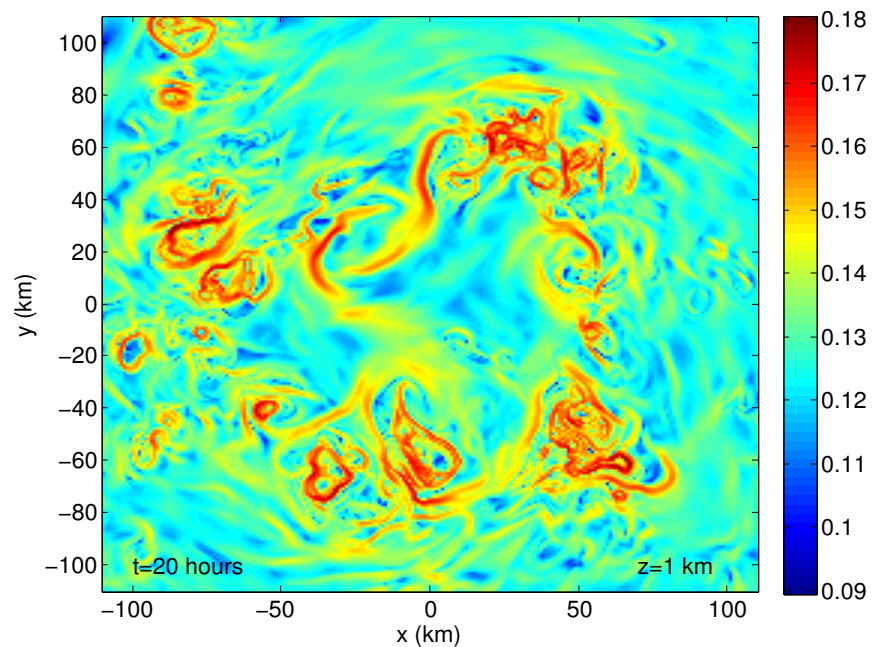

(b)

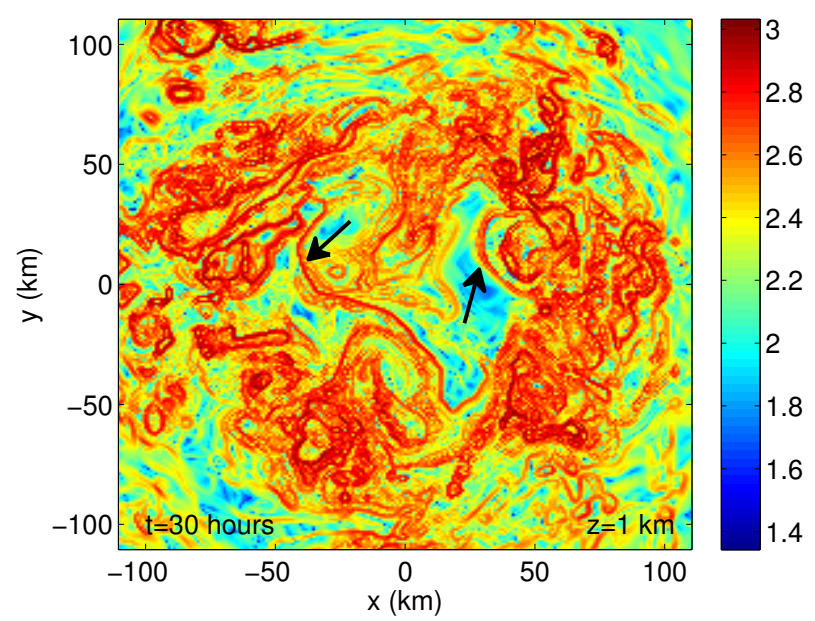

(d)

Fig. 4. LCSs from the planar FTLE field are shown for integration times of $30 \mathrm{~min}(\mathbf{a}), 1 \mathrm{~h}(\mathbf{b}), 2 \mathrm{~h}(\mathbf{c})$, and $4 \mathrm{~h}$ (d). Arrows show examples of LCSs that are visible under all shown integration times.

\subsection{Vertical LCS structure and convection}

The organizational aspects of the flow field and associated VHTs can be further illustrated by plots of particle trajectory locations in relation to regions of enhanced convection and LCSs. In Fig. 5, we show the initial locations of three small grids of particles overlaid on the LCSs at $30 \mathrm{~h}$ at $z=1 \mathrm{~km}$ and near the sea-surface in (a) and (b), respectively. Particles are seeded in three regions, a region of a tangle of FTLEs (black), a region containing low FTLE values that is located between a stable and unstable manifold segment (blue), and a region of low FTLE values that is not enclosed by intersecting LCSs (red). The final three-dimensional locations from trajectories initialized at $z=1 \mathrm{~km}$ height and near the sea surface are shown in (c) and (d), respectively. The final locations of the set initiated in the tangle show a large vertical displacement and particle dispersion in the upper troposphere, while the two sets in regions of low FTLE values show little dispersion. The large particle dispersion is typical for particles seeded in a tangle. On the other hand, particles initiated in regions with low FTLE values do not experience convection under the chosen integration time, so the only regions where the flow is approximately two-dimensional is in regions with low particle dispersion. While distinct FTLE ridges are present as boundaries between regions that have little interaction and mixing, the FTLE tangles prove as useful markers of deep convection.

The behavior of particles in relation to LCSs can be further described by changing the integration times of a cluster of trajectories and the associated Lagrangian fields. In Fig. 6a, the FTLE field with a $4 \mathrm{~h}$ integration time is shown with three sets of initial particle locations. The set of particles marked in cyan are initially located in an FTLE tangle, but the tangle is not visible under a $1 \mathrm{~h}$ integration time (cf. Fig. 5a). 


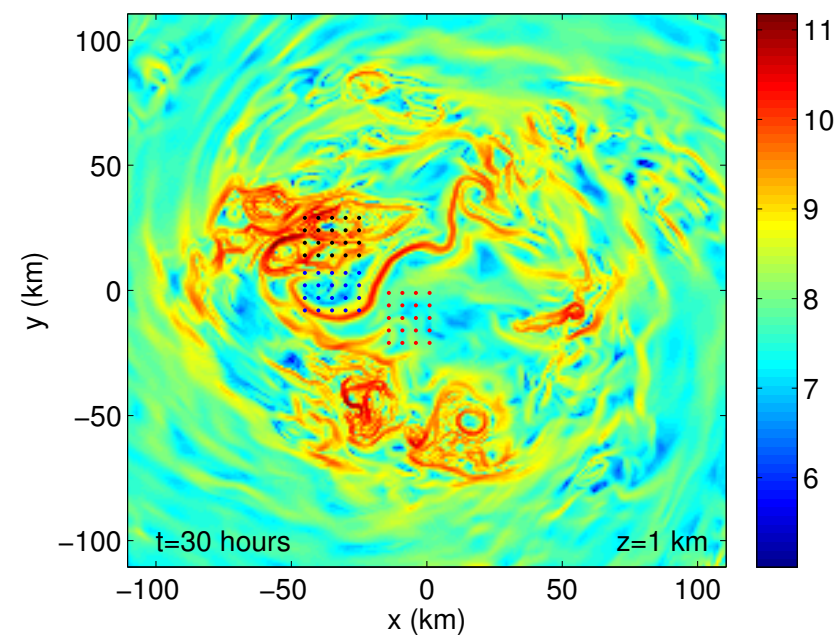

(a)

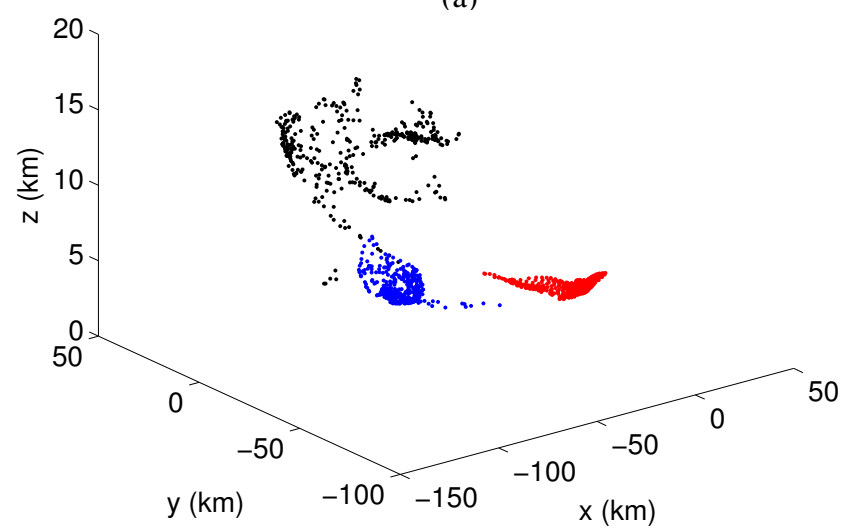

(c)

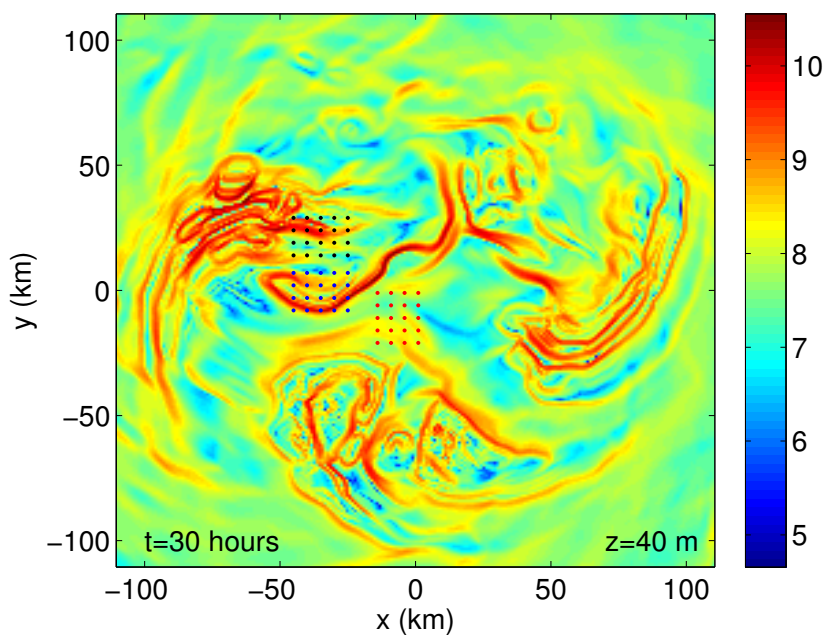

(b)

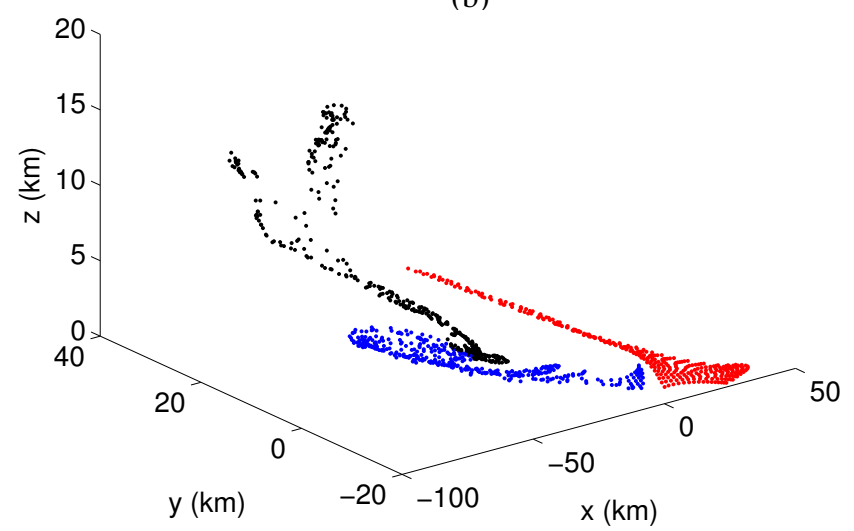

(d)

Fig. 5. LCSs from the planar FTLE field are shown at $z=1 \mathrm{~km}$ (a) and at $z=40 \mathrm{~m}$ (b) with initial particle locations shown as blue, red and black dots. The final locations of the particles after a $1 \mathrm{~h}$ integration are shown in (c) and (d), respectively.

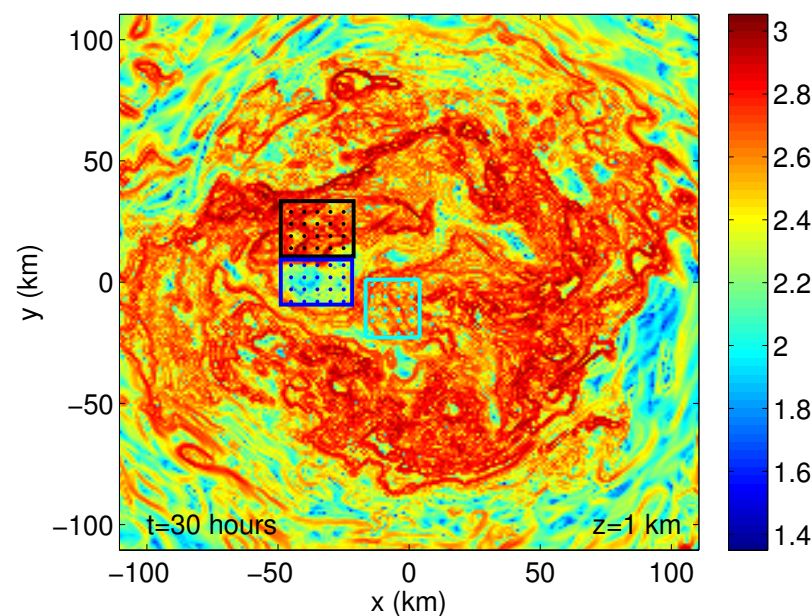

(a)

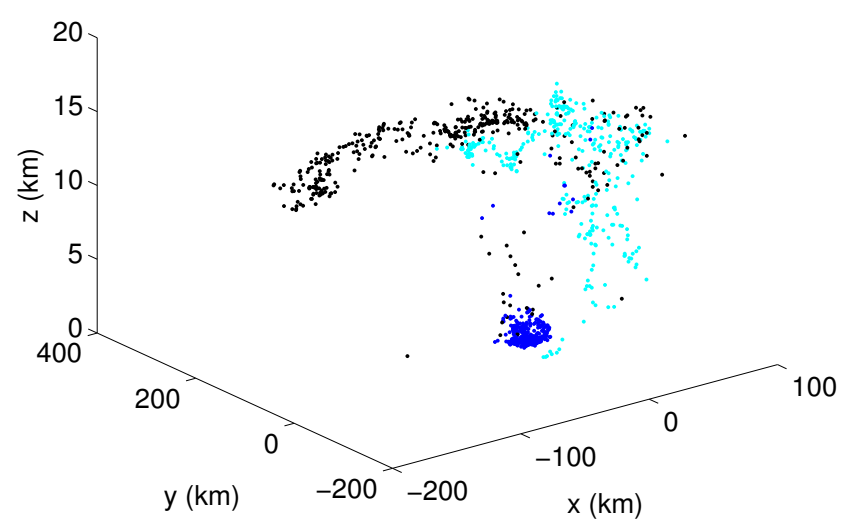

(b)

Fig. 6. LCSs from the planar FTLE field are shown at $z=1 \mathrm{~km}$ (a) with initial particle locations shown as blue, cyan and black dots. The final locations of the particles after a $4 \mathrm{~h}$ integration are shown in (b). 


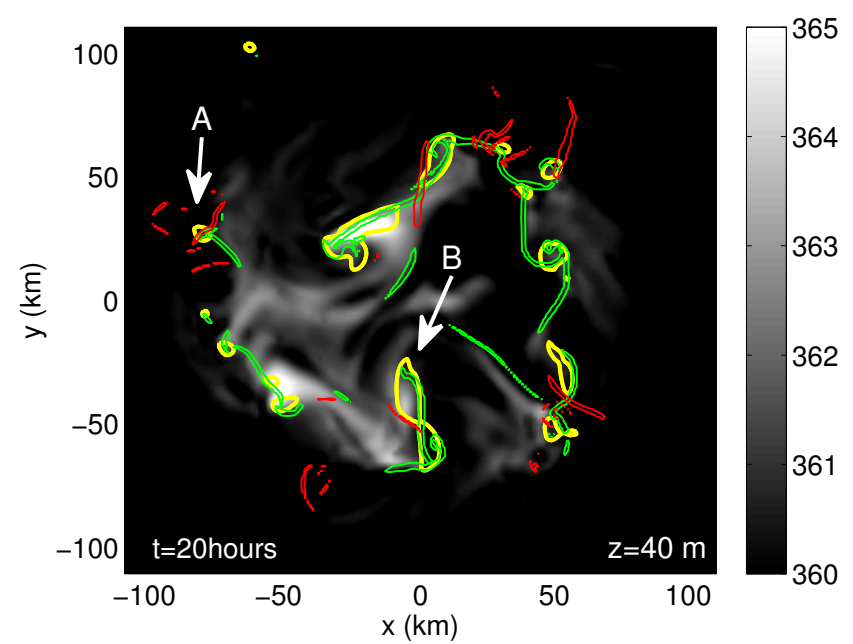

(a)

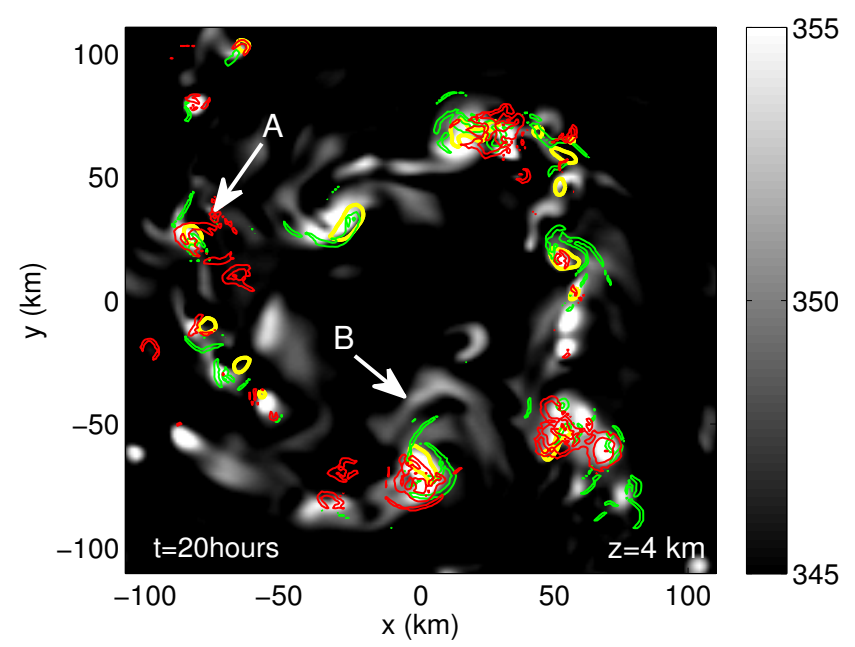

(c)

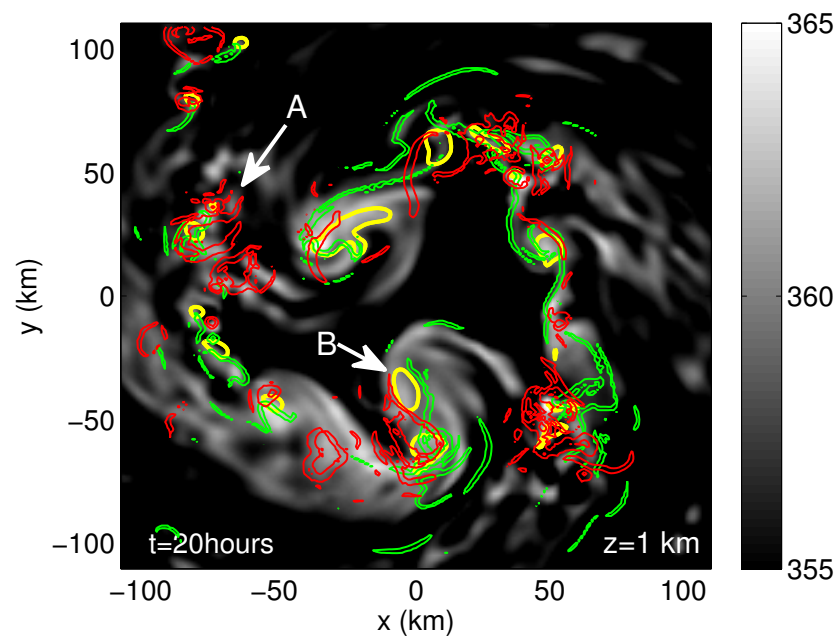

(b)

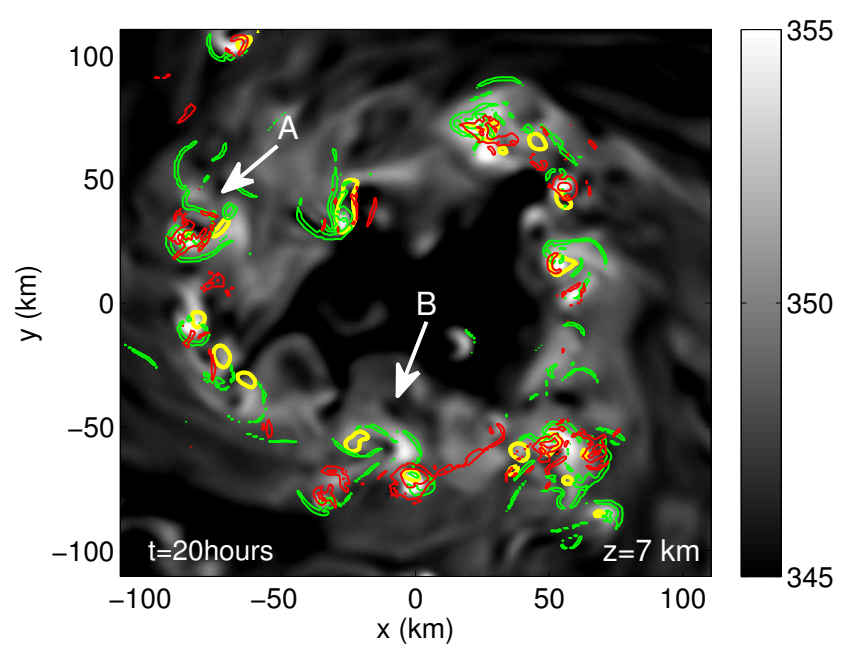

(d)

Fig. 7. The vertical structure of the LCSs are shown by attracting LCSs (green) and repelling LCSs (red), which with vorticity contours (yellow) are all overlaid on the $\theta_{\mathrm{e}}$ field (black and white shading) at vertical levels of $40 \mathrm{~m} \mathrm{(a),} 1 \mathrm{~km}(\mathbf{b}), 4 \mathrm{~km}(\mathbf{c})$, and $7 \mathrm{~km}(\mathbf{d})$.

The final behavior of these trajectories under a $4 \mathrm{~h}$ integration time (shown in cyan in Fig. 6b) is similar to another set of particles (black, Fig. 5a, c) which were also initialized in a tangle. However, a third set of particles (blue, Fig. 6a) initiated between the intersecting manifolds still maintains little dispersion after a $4 \mathrm{~h}$ integration time (Fig. 6b).

Convective vortices exhibit vertical coherence of Lagrangian fields as well as vorticity and $\theta_{\mathrm{e}}$. In Fig. 7, we show an example of Lagrangian fields and vorticity overlaid on $\theta_{\mathrm{e}}$ at levels of $40 \mathrm{~m}, 1 \mathrm{~km}, 4 \mathrm{~km}$, and $7 \mathrm{~km}$. Many pools of vorticity can be seen extending from the sea surface to heights of $7 \mathrm{~km}$ and higher, showing the vertical coherence associated with vortex tube stretching in a rotating environment. The pools of high $\theta_{\mathrm{e}}$ are in similar planar locations as vorticity maxima from the sea-surface to $4 \mathrm{~km}$ height, and to $7 \mathrm{~km}$ height in the smaller-radius VHTs.
The signature of deep convection in low-level FTLE fields can be seen further by comparing the FTLE-fields at $1 \mathrm{~km}$ in Fig. $7 \mathrm{~b}$ with the $\theta_{\mathrm{e}}$ fields at $7 \mathrm{~km}$ in Fig. $7 \mathrm{~d}$. The figures show that $\theta_{\mathrm{e}}$ at upper levels is coincident with high FTLE values at low levels. Moreover, the most intense convection is coincident with greater vertical coherence of the tangle, which suggests that trajectories remain entrained through convection. As an example, we show two specific vortices with different vertical characteristics marked A and B in Fig. 7. Vortex A has vertically coherent LCSs and is convectively active to $7 \mathrm{~km}$. Vortex B has no LCSs at $4 \mathrm{~km}$, and elevated vorticity and $\theta_{\mathrm{e}}$ does not extend to $4 \mathrm{~km}$. 


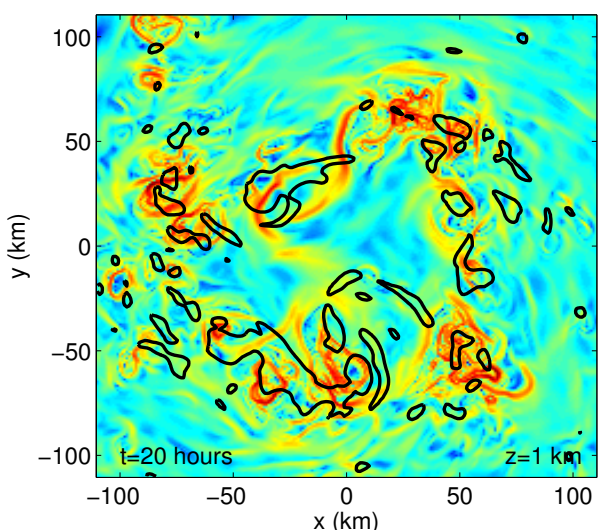

(a) FTLE's

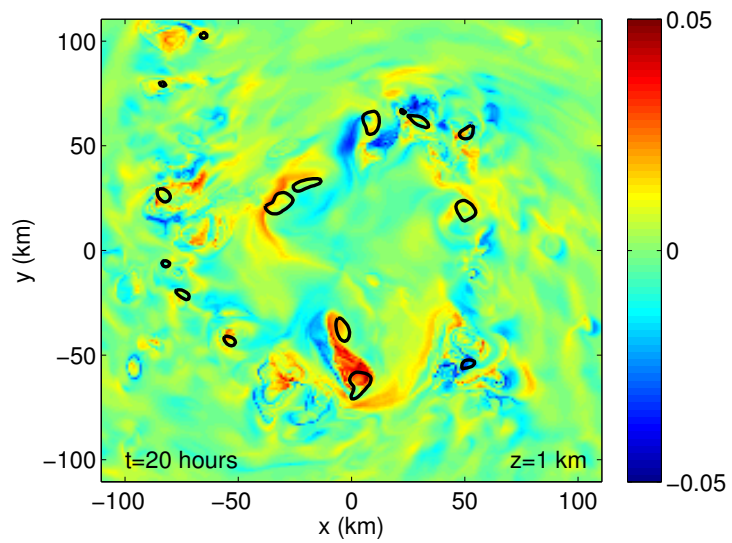

(c) $\Psi_{22}$

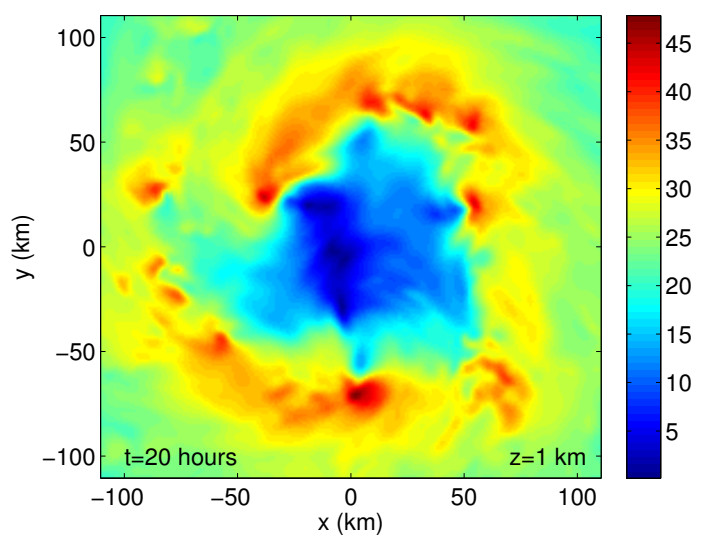

(e) $v_{t a n}$

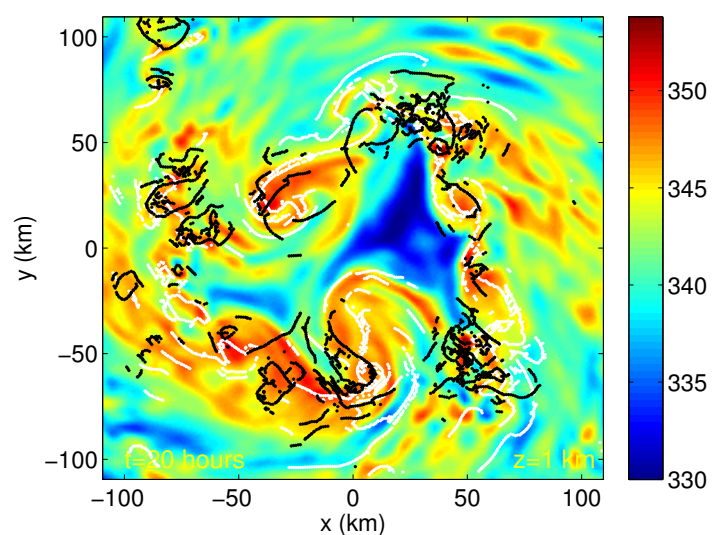

(b) $\theta_{e}$

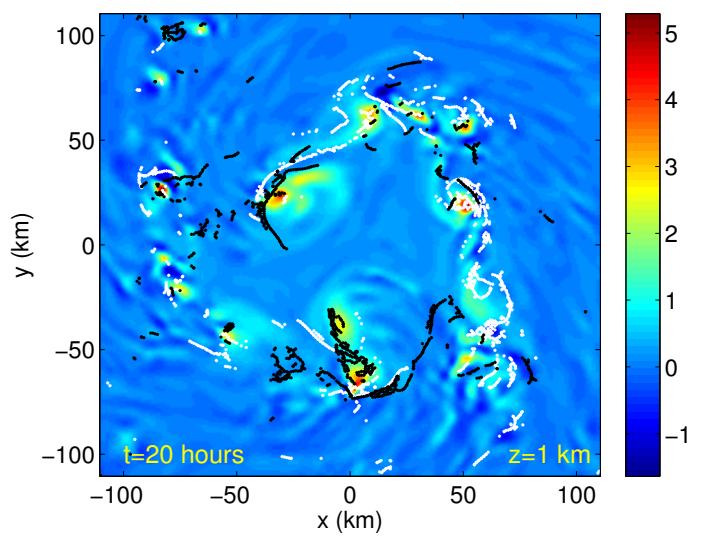

(d) vorticity

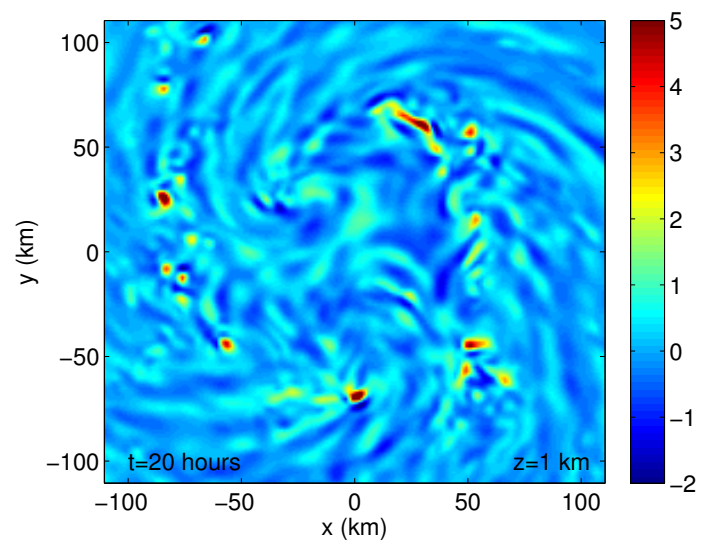

(f) $\mathrm{w}$

Fig. 8. Horizontal slices of Lagrangian fields and Eulerian diagnostics are compared at $20 \mathrm{~h}$ and $1 \mathrm{~km}$ height. The FTLE field (a) has ridges, which are extracted and overlaid on the $\theta_{\mathrm{e}}$ field (b). The $\Psi_{22}$ field (c) has ridges, which are extracted and overlaid on the $\zeta$ field (d). Tangential winds and vertical velocity are shown in (e) and (f).

\subsection{Horizontal structure}

\subsubsection{Azimuthal average}

To compare the aggregate contributions of the Lagrangian quantities to those of Eulerian quantities, e.g., vorticity and $\theta_{\mathrm{e}}$, we show the radius-time portrayal of several diagnostics in Fig. 2. The evolution of azimuthally averaged Lagrangian fields (FTLEs and $\Psi_{22}$ ) indicate that higher Lagrangian values are broadly coincident both radially and temporally with higher wind speeds, and higher $\theta_{\mathrm{e}}$. While the distribution of wind speed maxima is radially diffuse, the Lagrangian maxima are more localized. The radial location of $\Psi_{22}$ maxima 


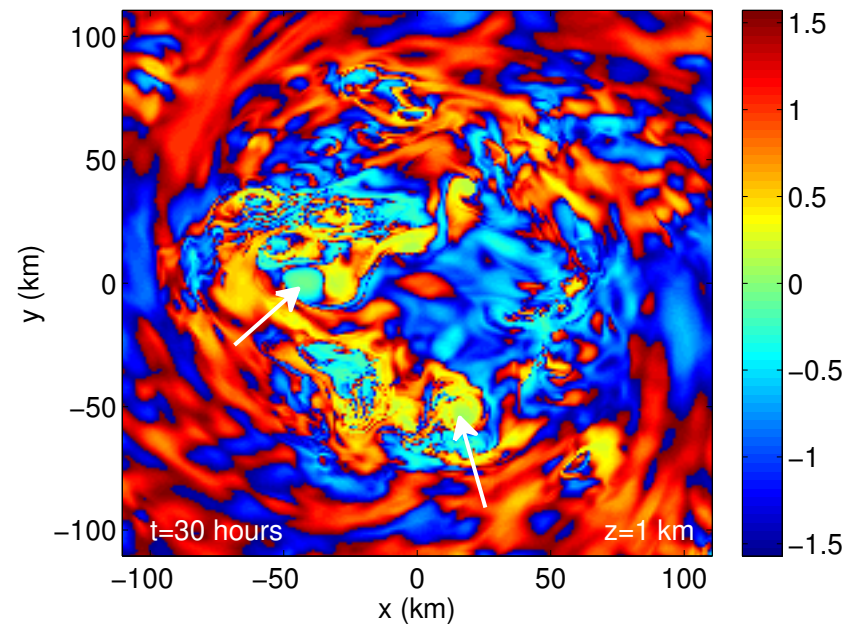

(a)

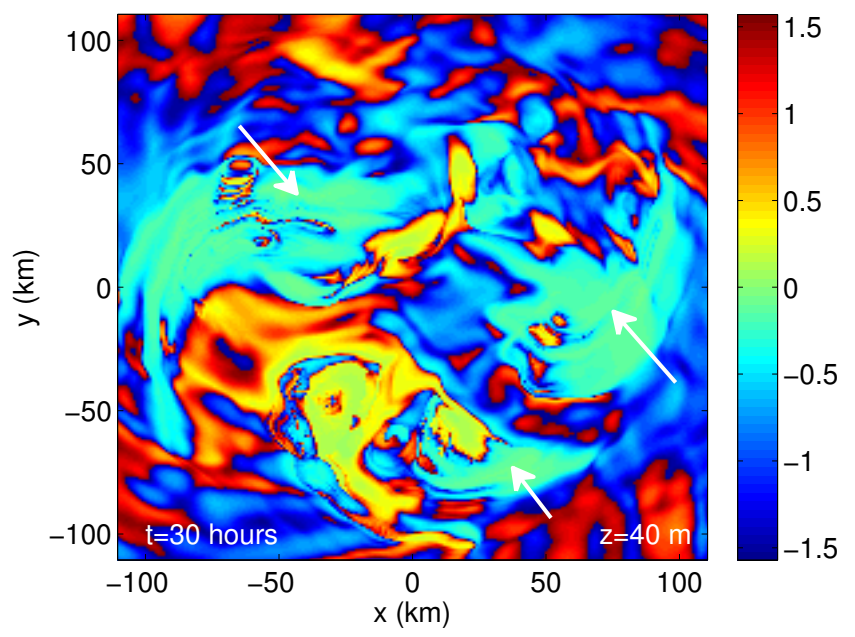

(b)

Fig. 9. $\varphi$ is shown for a $1 \mathrm{~h}$ integration time at $t=30 \mathrm{~h}$ at $z=1 \mathrm{~km}$ (a) and $z=40 \mathrm{~m}$ (b). Arrows indicate regions of Lagrangian neutral stability.

are near the inner core vortex edge where vorticity is maximized, Fig. 2e, f, while FTLEs are large throughout the region of high radial shear and convective updraughts, Fig. 2d. While vorticity mixes toward the origin, $\Psi_{22}$ does not, as it is associated with the eyewall boundary. Comparing the vorticity fields and $\Psi_{22}$ fields (Fig. 8c, d), shows that while the maxima occur at similar radii, they generally occur at different azimuths. As will be discussed in Sect. 5.2, the $\Psi_{22}$ ridges will be suggested to be important for the merging of vortical remnants, and they tend to occur near the largest regions of high vorticity, even during the intensification phase.

\subsubsection{Coherent Lagrangian vortices}

Coherent Lagrangian vortices reside where regions of Lagrangian neutral stability coincide with enhanced vorticity. The $\varphi$-field shows regions of neutral stability, meaning little stretching or shear and a nearly shape-preserving flow, by $\varphi$ values near zero. Figure 9 shows the $\varphi$-field on two horizontal slices, at $z=1 \mathrm{~km}$ and at $z=40 \mathrm{~m}$ in (a) and (b) respectively. Arrows mark examples of regions with nearly neutral Lagrangian stability. At $z=1 \mathrm{~km}$, there are some isolated regions of nearly neutral stability which coincide with vortex locations. In particular, a coherent vortex is present where the sets of trajectories with little dispersion were initiated in Figs. 5a and 6a. At the same planar location but at $z=40 \mathrm{~m}$, Fig. 9b, the $\varphi$-field still shows neutral stability.

\subsection{Vortex flow geometry}

We now consider the finite-time planar 2-D interactions of a pair of vortices in a time-dependent flow. Individual vortices often occur as cyclonic anticyclonic dipoles. While anticyclonic vortices arise through the tilting of horizontal vorticity into the vertical direction and subsequent stretching in updraught regions, their smaller strength, and thus smaller signature in the Lagrangian flow, makes their associated flow boundaries difficult to detect. To conceptualize the planar flow geometry, we restrict our view to the merging of a pair of cyclonic vortices, and their relation to LCSs nearby.

Two nearby vortices are often connected by an LCS that resides between them (Lapeyre, 2002). The mixing between two vortices can be described through the lobe dynamics of the stable and unstable manifolds of a saddle point that is located between them, and was studied in an idealized configuration involving just two vortices by Velasco Fuentes (2001). For shorter integration times, the LCSs are not long enough to describe the mixing through lobe dynamics. However, the finite-time interactions can still be described by the LCSs. Here we present a heuristic classification of the interaction of cyclonic vortices (VHTs or their remnants) into stable, unstable, or non-interaction based on the type of LCSs between them.

\subsubsection{Stable interaction}

Stable vortex interaction as depicted in Fig. 10b occurs when the influence of the repelling LCS is greater than that of the attracting LCS. This situation is most often observed with vortical remnants of VHTs and $\boldsymbol{\Psi}$-LCSs. A repelling $\boldsymbol{\Psi}$-LCS appears between adjacent vortices while an attracting LCS does not appear. The flow direction for cyclonic vortices associated with the system-scale circulation dictates the connection of the LCS to be radially inward of the leading vortex and radially outward of the trailing vortex. The vortices move toward the center of the LCS, but do not mix since they remain separated by the LCS. Because of the general in, up and out circulation driven by the aggregate heating of the VHTs, the vortices at low-levels have a tendency to move radially 


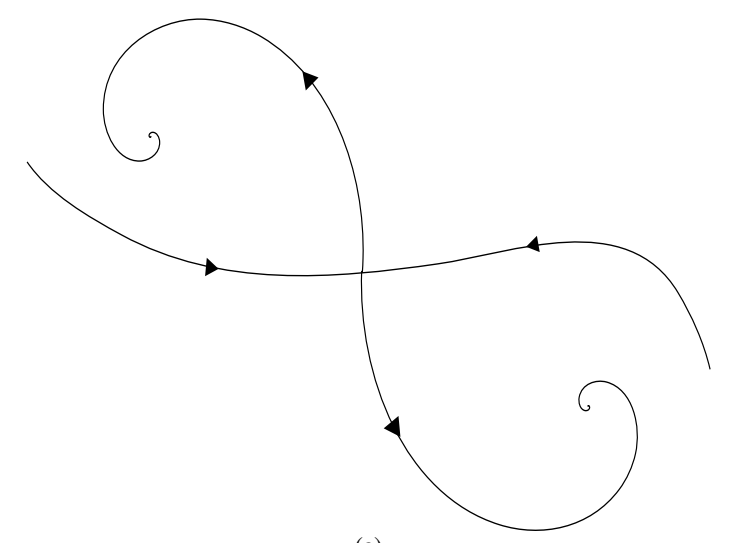

(a)

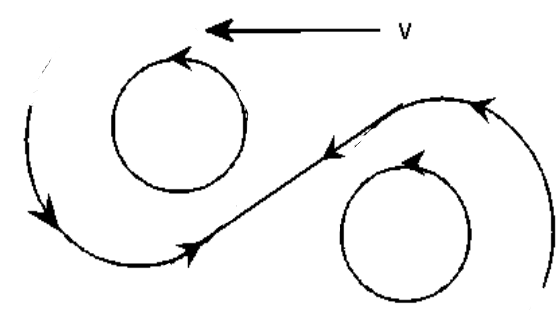

(b)

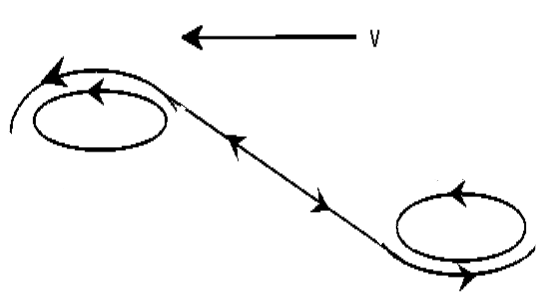

(c)

Fig. 10. A pair of convergent vortices on opposing sides of a saddle are kept from finite-time interaction (a). Stable (b) and unstable (c) vortex interactions occur along a single manifold segment when the other manifold branch is not obtained under short integration times.

inward in the absence of flow boundaries, and often merge after the LCS vanishes.

\subsubsection{Unstable interaction}

Unstable vortex interaction as depicted in Fig. 10c is the basic type of interaction for convergent vortices along attracting LCSs, which tend to horizontally stretch VHTs or their remnants. The formation of an attracting LCS between two adjacent VHTs forces the orientation to be opposite the case for stable vortex interaction. As the vortices are attracted to the LCS, the vortex tends to move toward the tail of the LCS, and to be stretched. Unstable vortex interaction is most noticable in the boundary layer where the attracting LCSs are enhanced and the repelling LCSs are reduced by the mean inflow, although it is also seen above the boundary layer.

\subsubsection{Non-interaction}

Vortex noninteraction, Fig. 10a, occurs over short times when both attracting and repelling LCSs intersect between vortices, and form a boundary which completely separates the vortices. Since high time-dependence limits the length of the LCSs and the vertical velocity component adds a degree of freedom to particle paths, vortex non-interaction does not induce lobe dynamics, and lasts for at most a few hours. Pairs of vortices that are separated by an LCS can be drawn arbitrarily close without merging during finite-time integrations of $2 \mathrm{~h}$ or less, in contrast to the results of Melander et al. (1988) for two-dimensional nondivergent vortex dynamics.

\subsubsection{An illustrative example of vortex and LCS inter- action}

A case of interacting vortices can be seen in Fig. 11 during the time period from $29 \mathrm{~h}$ to $31 \mathrm{~h}$. At $29 \mathrm{~h}$, there are initially 3 prominent vorticity pools in the lower troposphere, labelled A, B, and C in Fig. 11a which form part of a pentagonal structure. A saddle point (in the frame of reference moving with the vortex pair and seen as the intersection of attracting and repelling LCSs) located between vortex A and vortex B creates a separatrix (in the planar flow) which divides the vortices, as in Fig. 10b. Over the next hour, all the vorticity pools and LCSs travel together, and at $30.4 \mathrm{~h}$, vortices A and B come very close, yet remain separated by the attracting LCS. The relationship between vortex B and vortex $\mathrm{C}$ is different, however, since they are separated by a single repelling LCS at $29 \mathrm{~h}$ and $29.8 \mathrm{~h}$ instead of an intersecting pair of LCSs. By $30.4 \mathrm{~h}$, the LCS vanishes and by $31 \mathrm{~h}$, vortices $\mathrm{B}$ and $\mathrm{C}$ begin to merge since there is no flow boundary separating them. These latter two vortices travel along the LCS and then merge just after $31 \mathrm{~h}$, as the vortex $\mathrm{C}$ absorbs vortex B. 


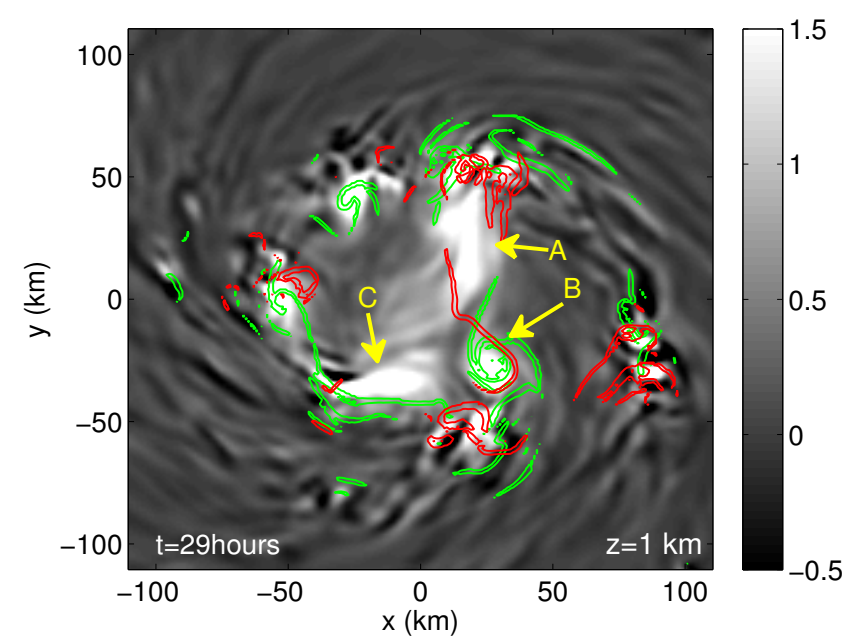

(a)

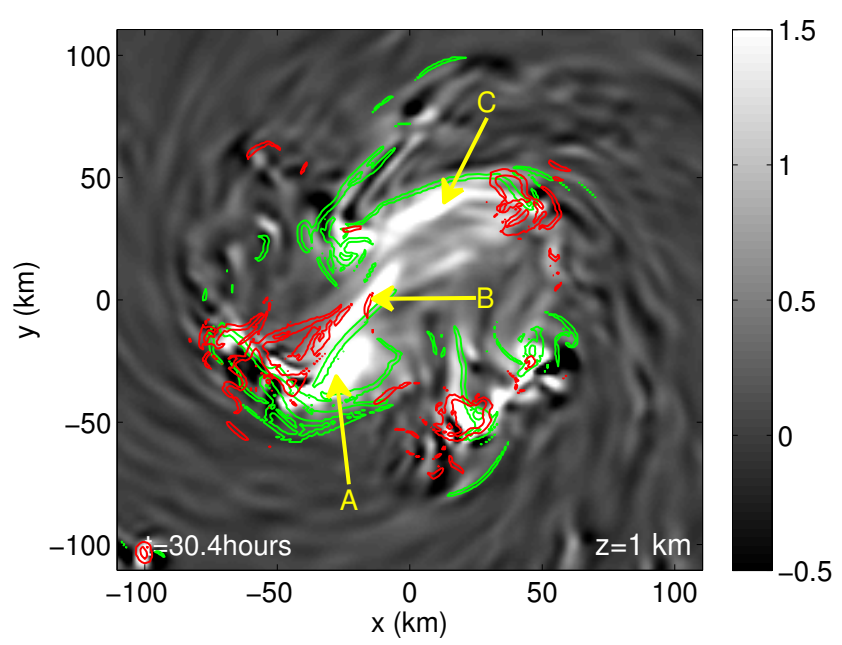

(c)

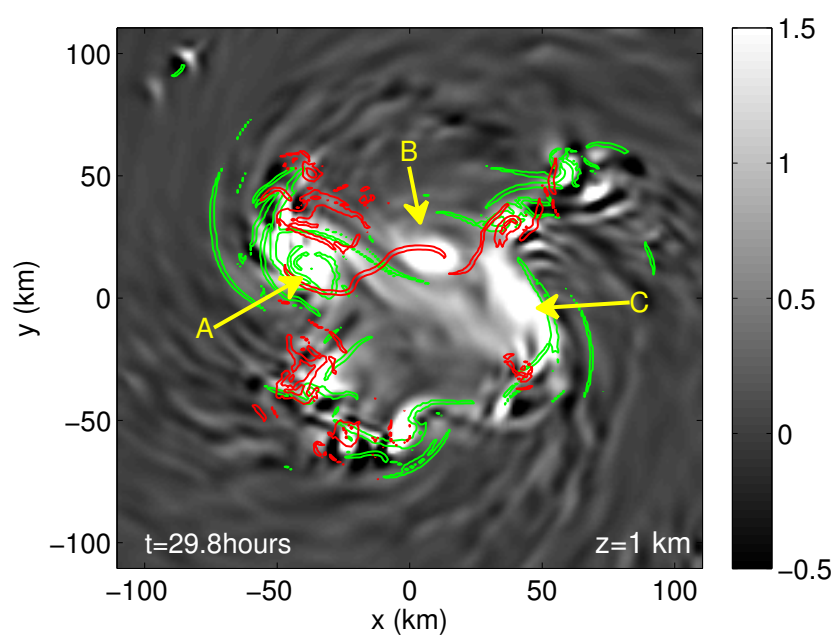

(b)

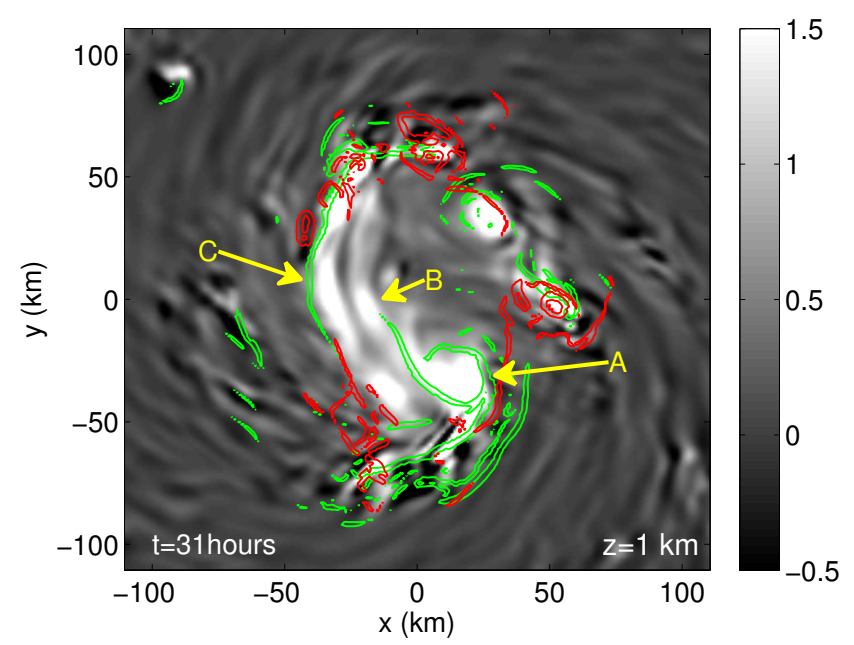

(d)

Fig. 11. An example of vortex interaction during the simulated intensification process. The times shown are $29 \mathrm{~h} \mathrm{(a),} 29.8 \mathrm{~h} \mathrm{(b),} 30.4 \mathrm{~h}$ (c), and $31 \mathrm{~h}$ (d). Arrows point to a subset of interacting vortices discussed in Sect. 4.5. The intense vorticity regions, attracting $\boldsymbol{\Psi}$-LCSs (green), repelling $\boldsymbol{\Psi}$-LCSs (red), are overlaid on the vorticity field $\left(\zeta \times 10^{-3} \mathrm{~s}^{-1}\right.$, black and white shading).

\section{Temporal evolution of VHTs and LCSs}

\subsection{Stirring of $\theta_{\mathrm{e}}$ in the boundary layer}

Having examined the interaction of intense vortices, we now consider the enhancement and distribution of the source of fuel for the vortices. Surface fluxes of moisture increase $\theta_{\mathrm{e}}$ at the bottom of the boundary layer, and $\theta_{\mathrm{e}}$ is drawn inward by the convergent circulation. In the absence of the import of dry air from aloft, $\theta_{\mathrm{e}}$ increases on inflow trajectories. Alongside the system-scale convergence, the LCSs reveal preferential locations for the convergence of $\theta_{\mathrm{e}}$ near the sea-surface, which subsequently leads to localized convection.

The evolution of boundary layer $\theta_{\mathrm{e}}$ can be seen in Figs. 12 and 13 by time snapshots at $2 \mathrm{~h}$ intervals of $\theta_{\mathrm{e}}$ along with vorticity contours and attracting and repelling coherent struc- tures at the lowest model output of $40 \mathrm{~m}$. A ring of high $\theta_{\mathrm{e}}$ near the initial VHT locations is transformed to a pool of high $\theta_{\mathrm{e}}$ in the center. At the same time, low $\theta_{\mathrm{e}}$ from the center diminishes as it is either expelled outward or enhanced by moisture fluxes in regions of air-sea disequilibrium. While $\theta_{\mathrm{e}}$ values increase radially inward, specific focal points of high $\theta_{\mathrm{e}}$ exist. When LCSs are seen, they primarily occur along $\theta_{\mathrm{e}}$ gradients. The $\Psi_{22}$-fields show similar attracting boundaries as the FTLEs. However, the repelling LCS tangles that mark regions of intense convection in the FTLE fields are largely absent in the $\Psi_{22}$-fields. Thus, the $\Psi$-LCSs better characterize the planar flow structure. Some $\theta_{\mathrm{e}}$ and vorticity pools can be tracked over the time interval shown, for example the largest vorticity pool in a region of high $\theta_{\mathrm{e}}$ is located at approximately $(0 \mathrm{~km},-50 \mathrm{~km})$ at $t=22 \mathrm{~h}$ in Figs. 12 and $13 \mathrm{~b}$. The vorticity and $\theta_{\mathrm{e}}$ pool can be seen at nearly the 


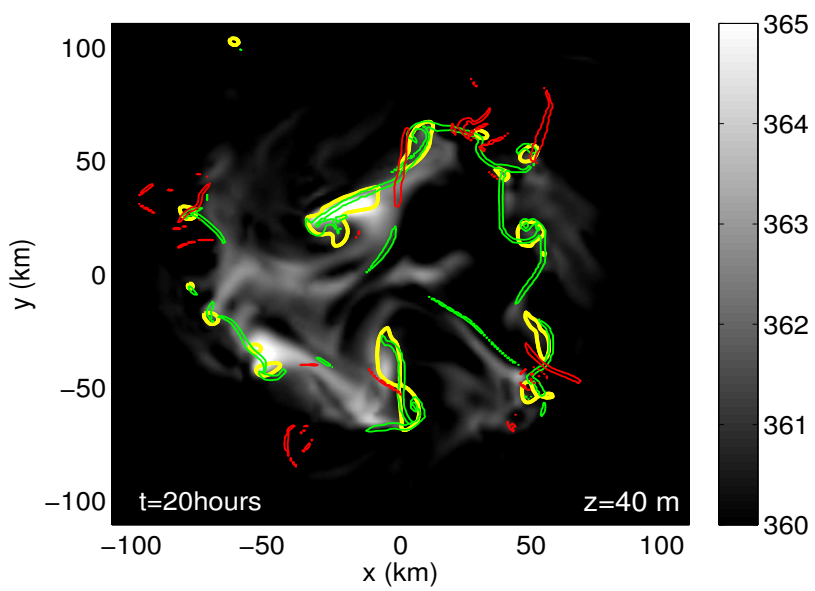

(a)

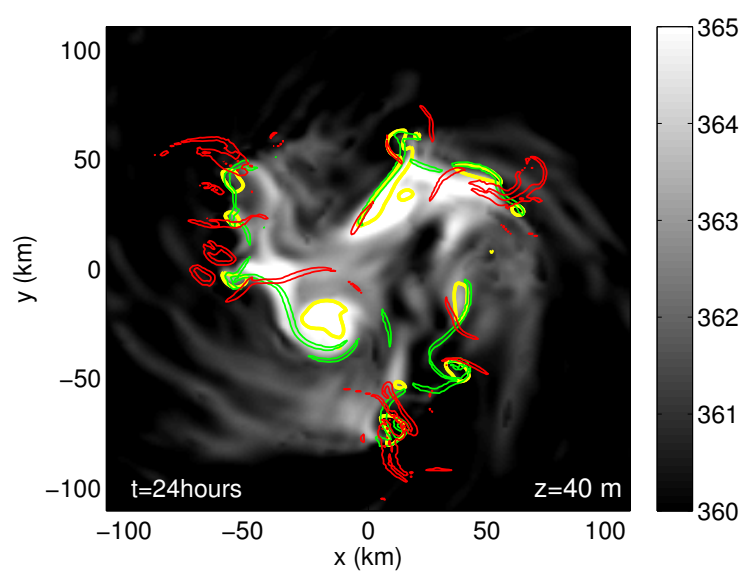

(c)

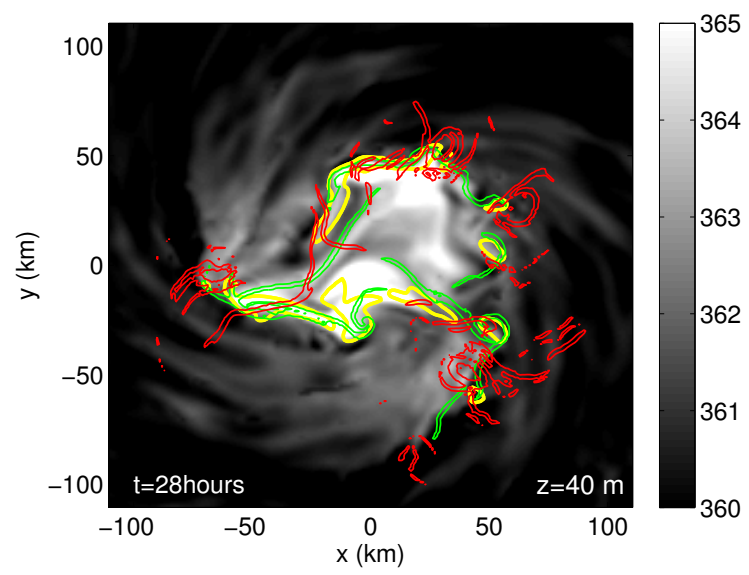

(e)

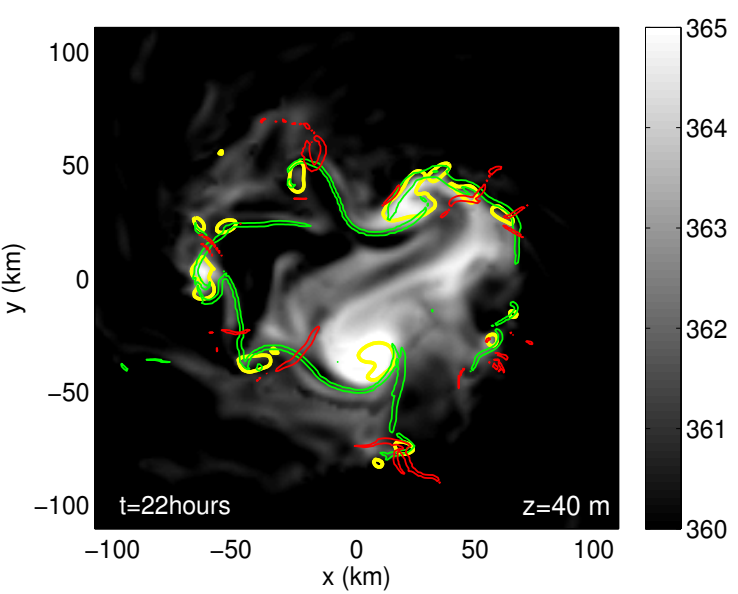

(b)

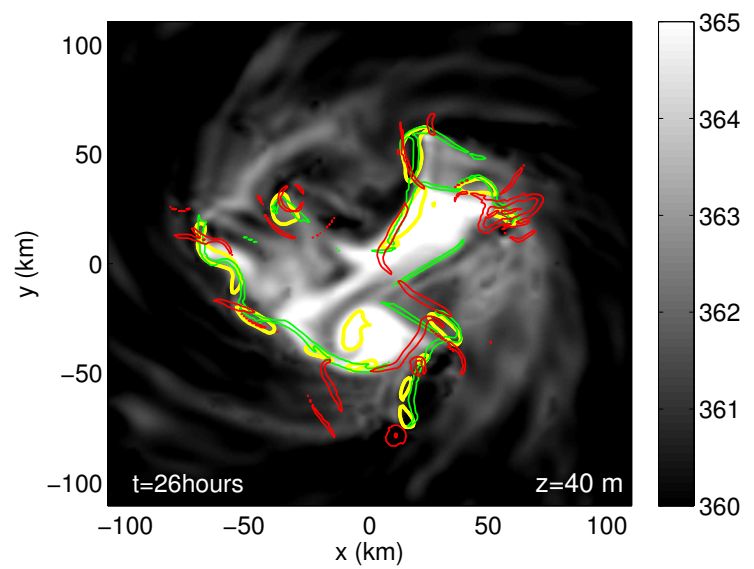

(d)

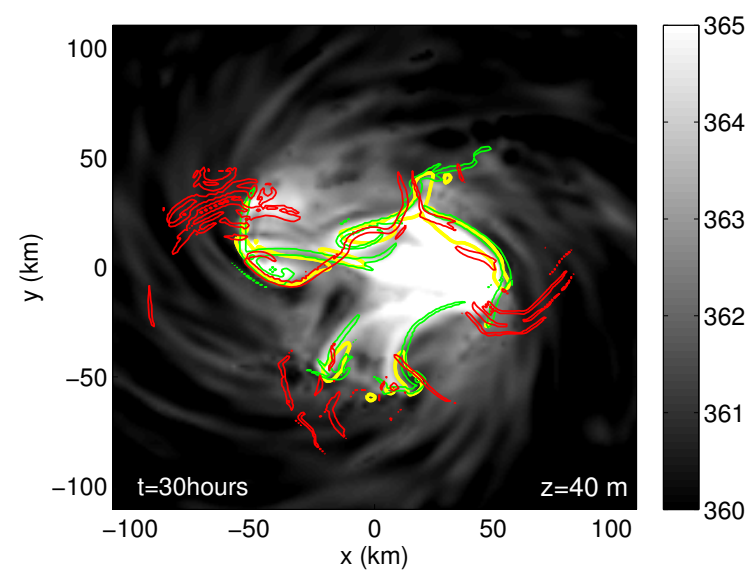

(f)

Fig. 12. Planar attracting (green) and repelling (red) FTLE ridges and vorticity contours (yellow) are overlaid on $\theta_{\mathrm{e}}$ fields with $1 \mathrm{~h}$ integration time at times from $20 \mathrm{~h}$ to $30 \mathrm{~h}$ every $2 \mathrm{~h}$ at the bottom of the boundary layer.

same location after rotating around the circulation center in Figs. 12 and 13, shown in panels (c) and (d) at $24 \mathrm{~h}$ and $26 \mathrm{~h}$, respectively. The attracting LCSs which are located alongside the vorticity pool at $22 \mathrm{~h}$ are still clearly visible at $26 \mathrm{~h}$. Other LCSs which at $20 \mathrm{~h}$ bound dry air in the center are still present at $30 \mathrm{~h}$, where they bound elevated $\theta_{\mathrm{e}}$ air near the vortex center. 


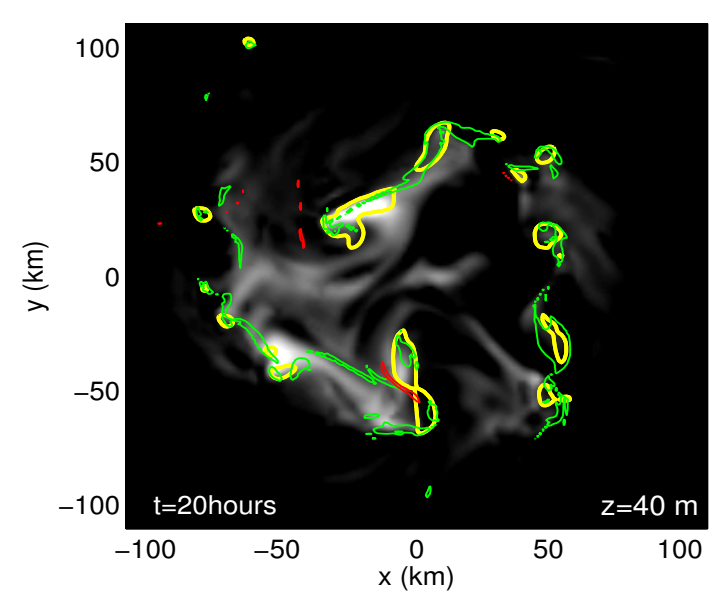

(a)

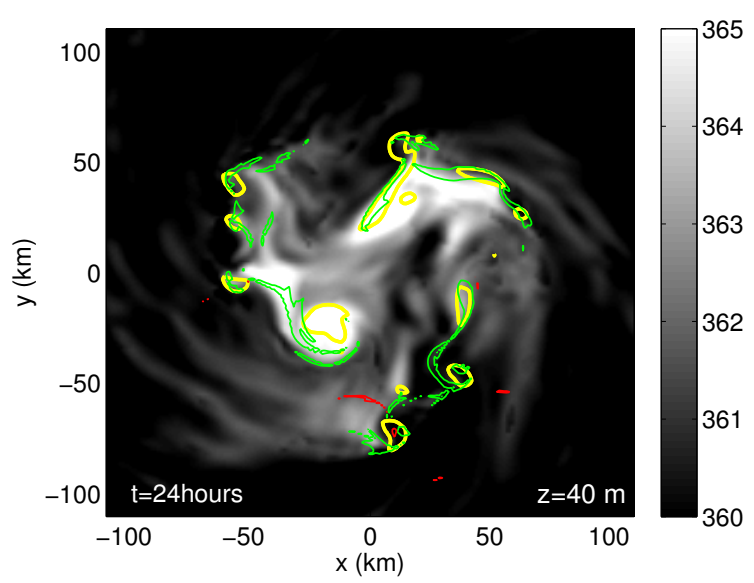

(c)

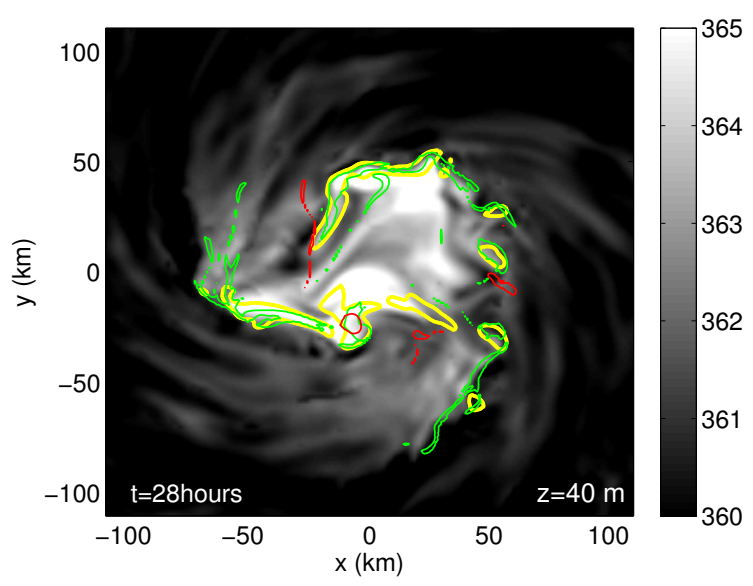

(e)
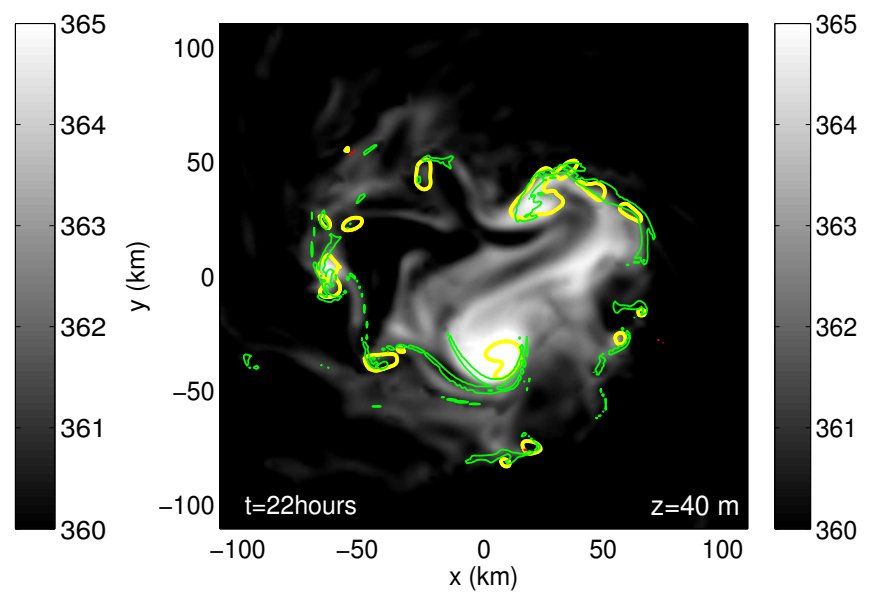

(b)

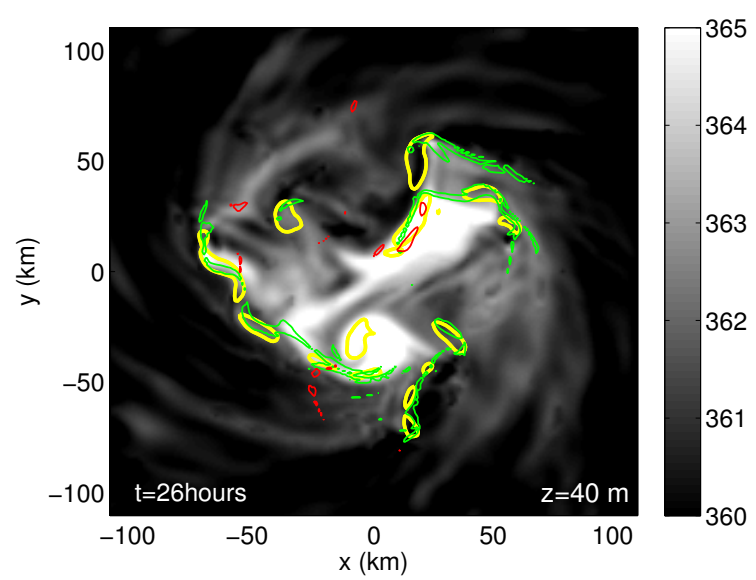

(d)

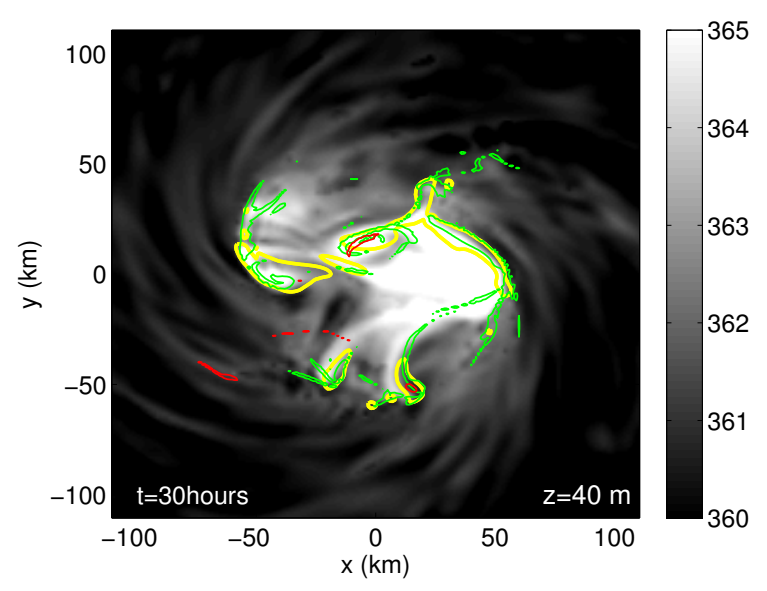

(f)

Fig. 13. Attracting (green) and repelling (red) $\Psi_{22}$ ridges and vorticity contours (yellow) are overlaid on $\theta_{\mathrm{e}}$ fields with $1 \mathrm{~h}$ integration time at times from $20 \mathrm{~h}$ to $30 \mathrm{~h}$ every $2 \mathrm{~h}$ at the bottom of the boundary layer.

\subsection{Organization of vortical remnants above the bound- ary layer}

Due to strong convergence, there are no isolated yet persistent hyperbolic repelling LCSs in the boundary layer. How- ever, above the boundary layer, these structures are seen with attracting LCSs and form the boundaries that describe vortex interaction there, shown in Figs. 14 and 15. The structure of the $\Psi_{22}$-field and FTLE-field is again similar along attracting 


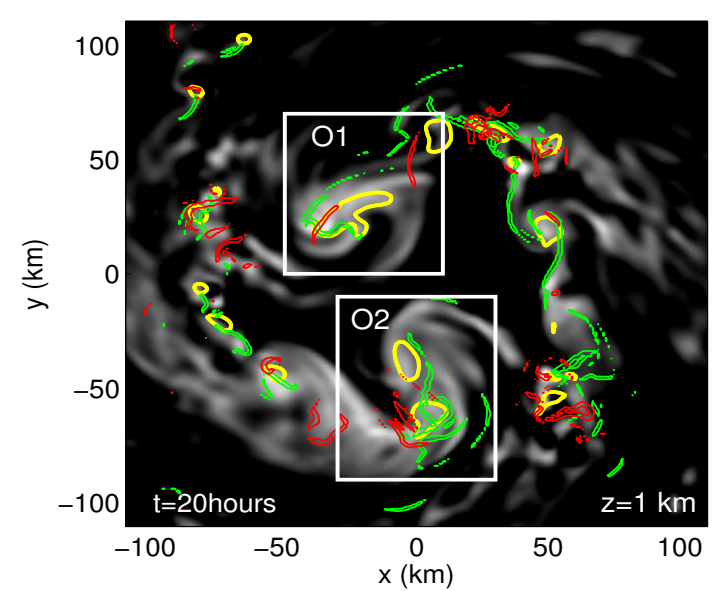

(a)

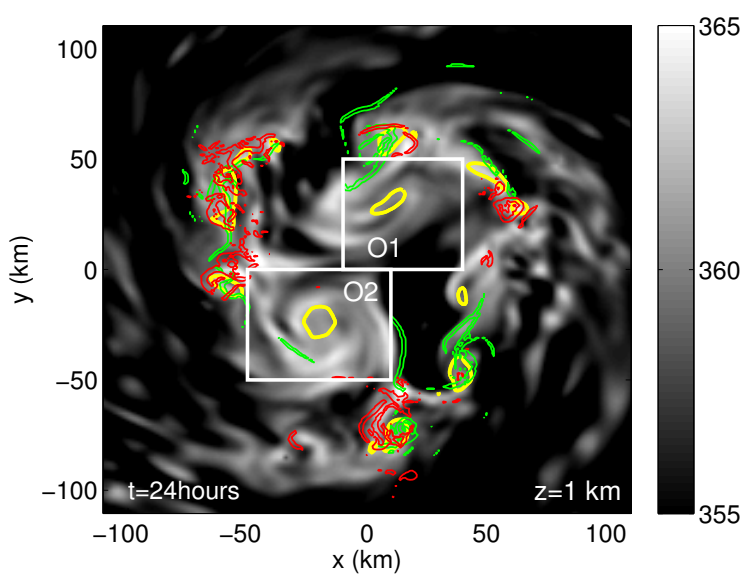

(c)

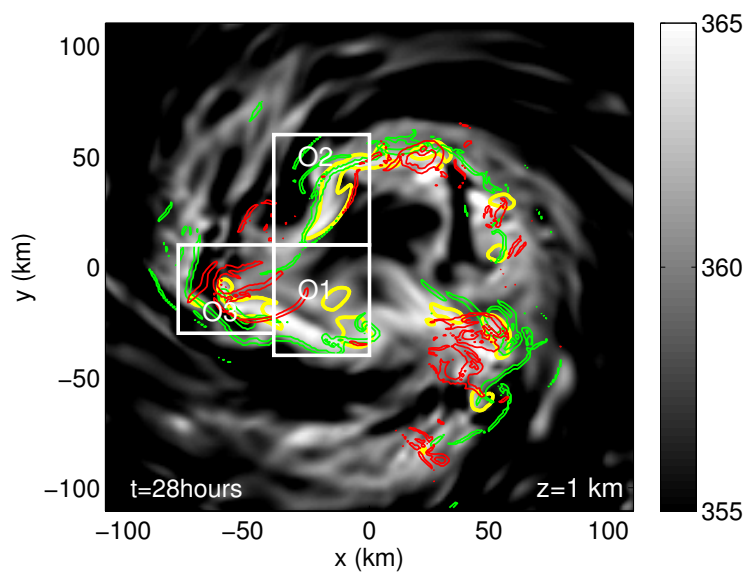

(e)
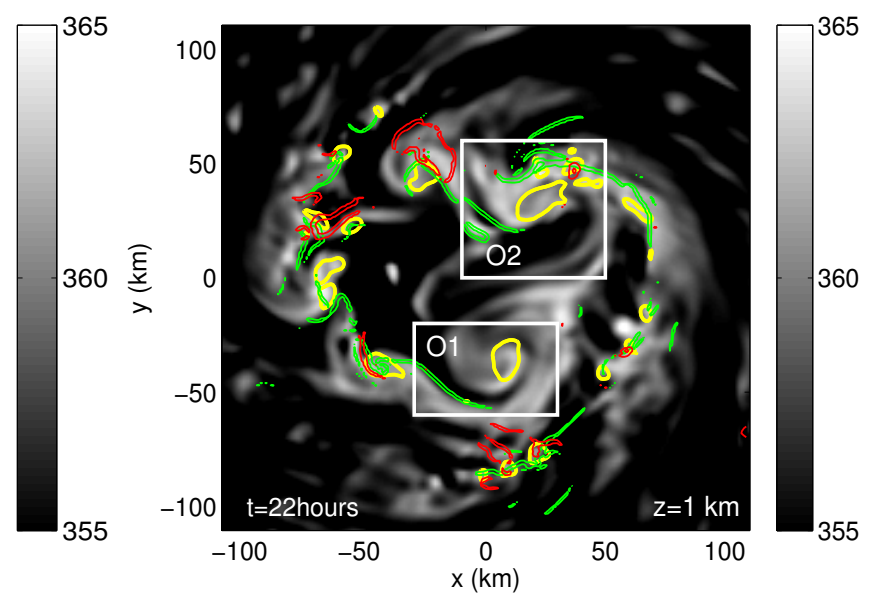

(b)

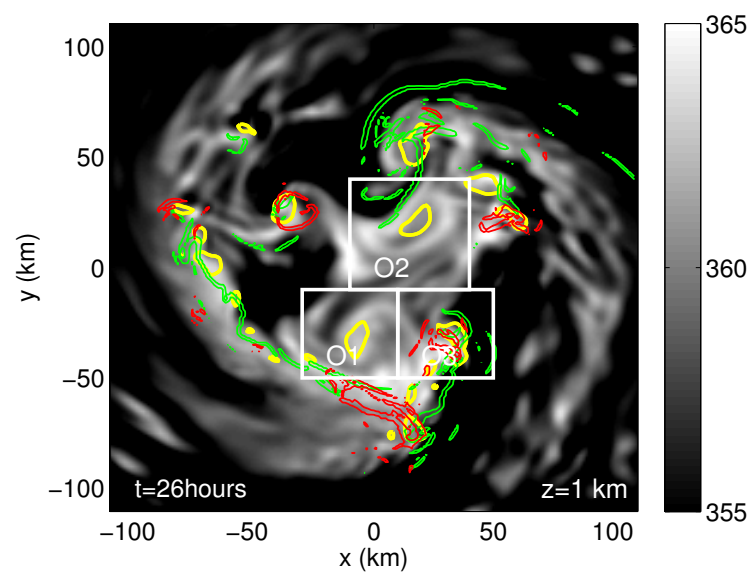

(d)

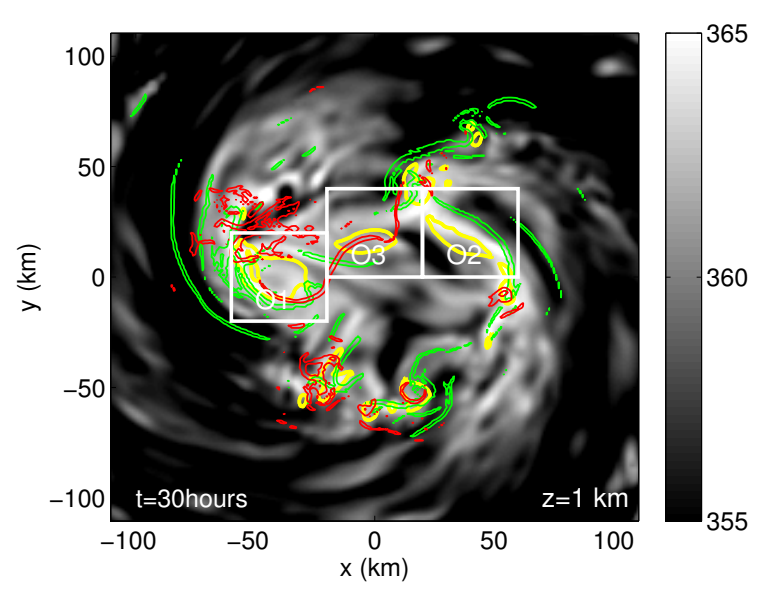

(f)

Fig. 14. Organized mixing regimes can be seen in labeled boxes containing FTLE LCSs at $z=1 \mathrm{~km}$ with $1 \mathrm{~h}$ integration time at times from $20 \mathrm{~h}$ to $30 \mathrm{~h}$ every $2 \mathrm{~h}$. The attracting LCSs (green), repelling LCSs (red), and vorticity contours (yellow) are all overlaid on the $\theta_{\mathrm{e}}$ field (black and white shading).

boundaries. However, the FTLEs again form tangles marking convection in the repelling LCSs. In contrast, coherent and trackable $\boldsymbol{\Psi}$-LCSs can be seen in several places without associated tangles.
We now examine the temporal evolution of the LCSs during the time period from $20-30 \mathrm{~h}$ at $1 \mathrm{~km}$ altitude which is near the top of the boundary layer associated with surface friction. We see that while the VHTs and $\theta_{\mathrm{e}}$ anomalies that 


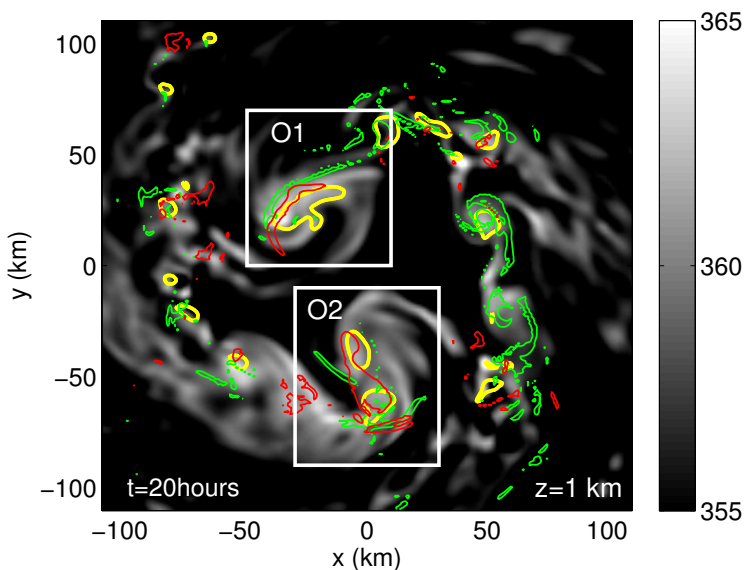

(a)

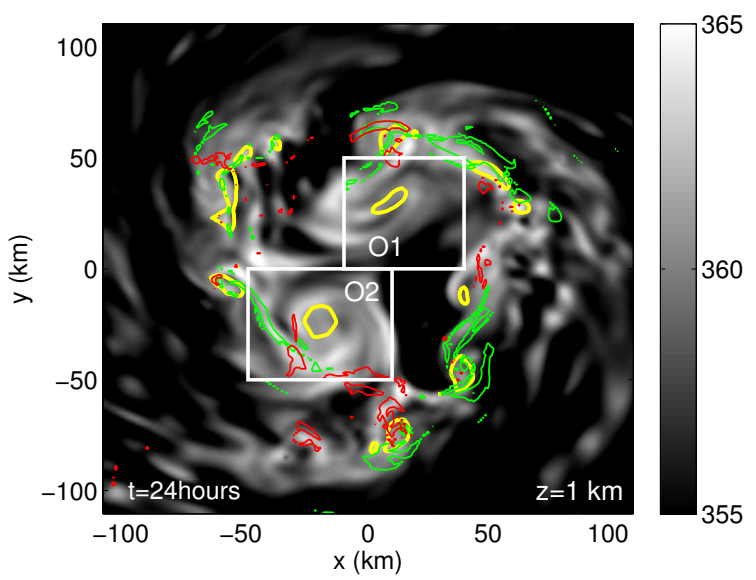

(c)

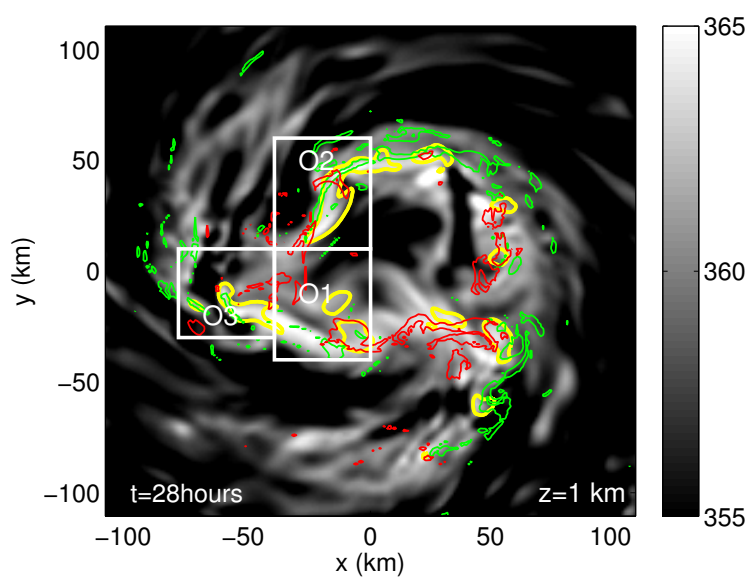

(e)

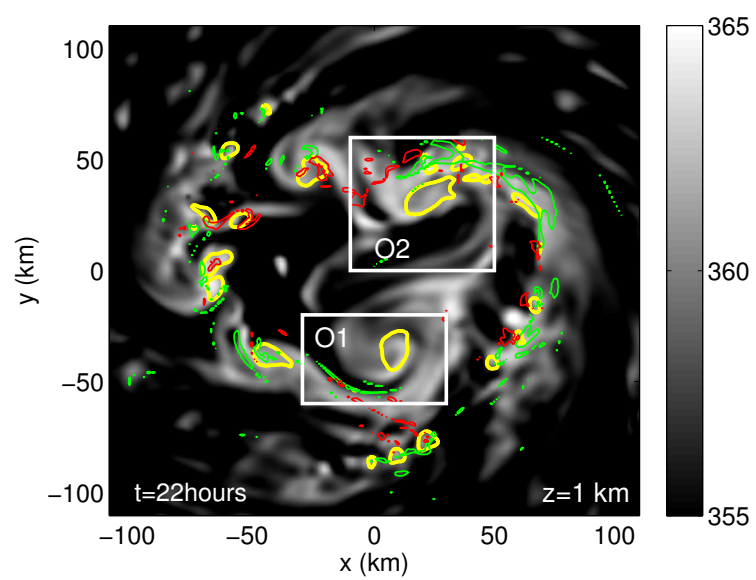

(b)

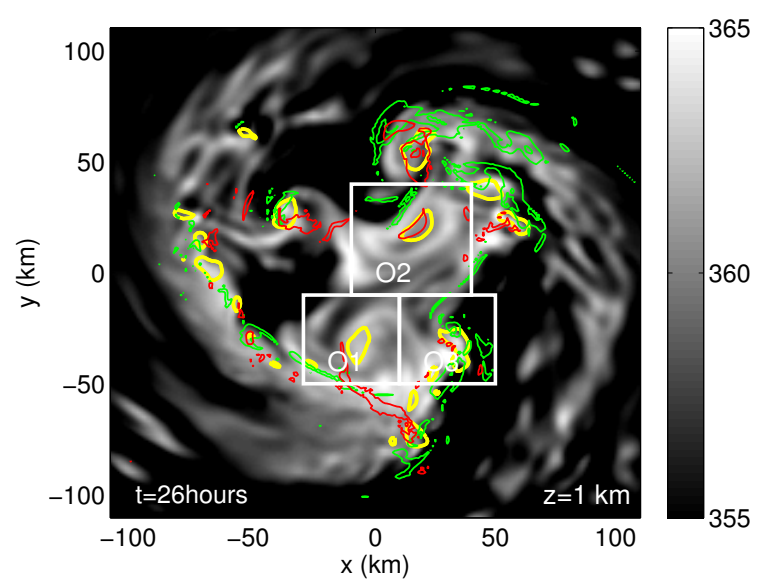

(d)

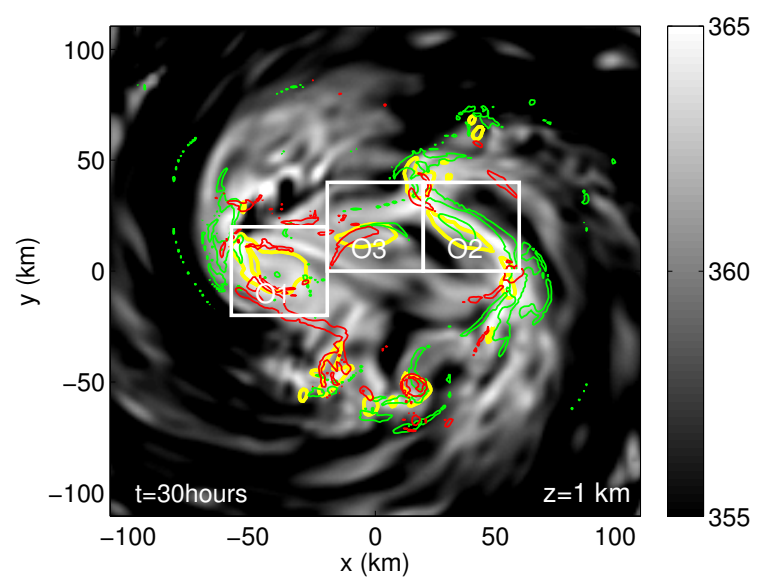

(f)

Fig. 15. Organized mixing regimes can be seen in labeled boxes containing $\Psi_{22}$ LCSs at $z=1 \mathrm{~km}$ with $1 \mathrm{~h}$ integration time at times from $20 \mathrm{~h}$ to $30 \mathrm{~h}$ every $2 \mathrm{~h}$. The attracting $\boldsymbol{\Psi}$-LCSs (green), repelling $\boldsymbol{\Psi}$-LCSs (red), and vorticity contours (yellow) are all overlaid on the $\theta_{\mathrm{e}}$ field (black and white shading).

support them create an environment of turbulent convection, the LCSs show an organized view of the inward transport of vortical remnants. Regions of "organized" mixing form LCSs which are longer lasting than other LCSs or individual
VHTs. Figures 14 and 15 show the time evolution of the flow field above the boundary layer and "organized" regions denoted by the "O" symbol and outlined by boxes. The regions are defined here as regions with coherent vorticity pools and 


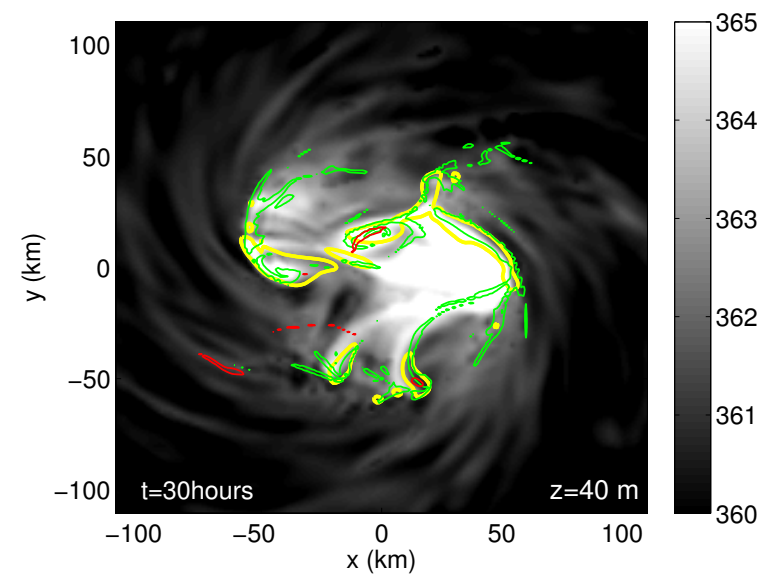

(a)

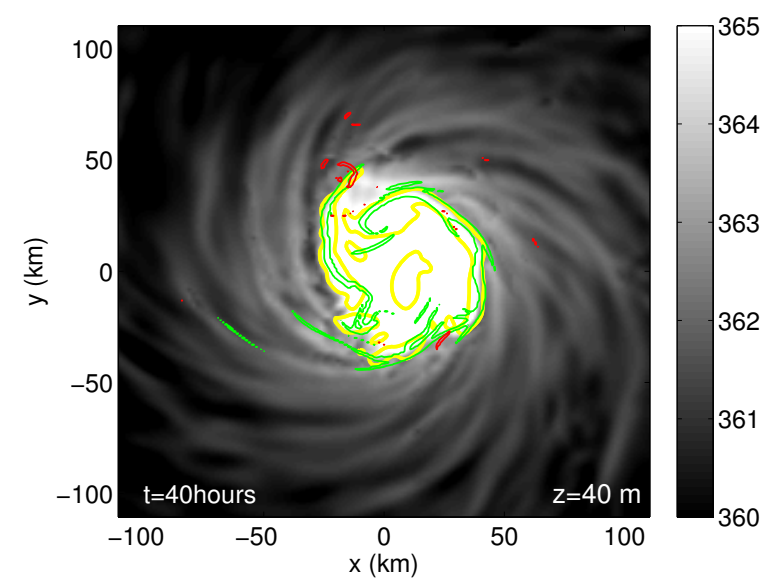

(c)

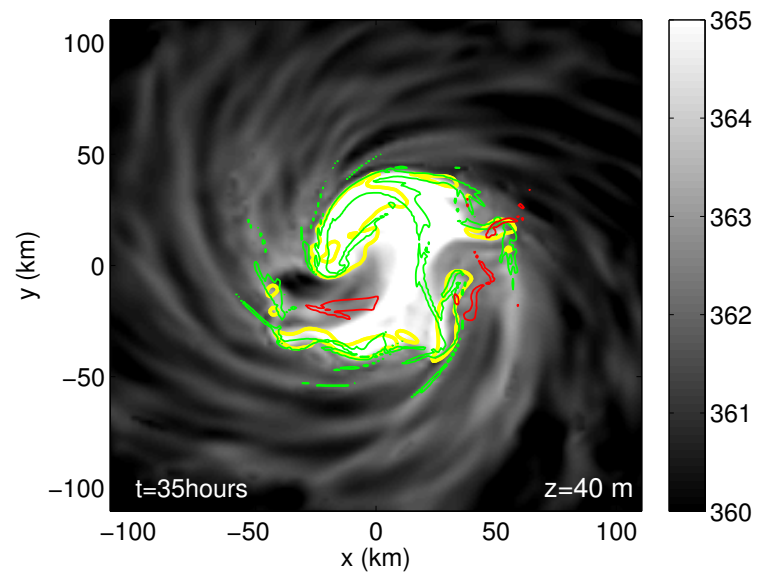

(b)

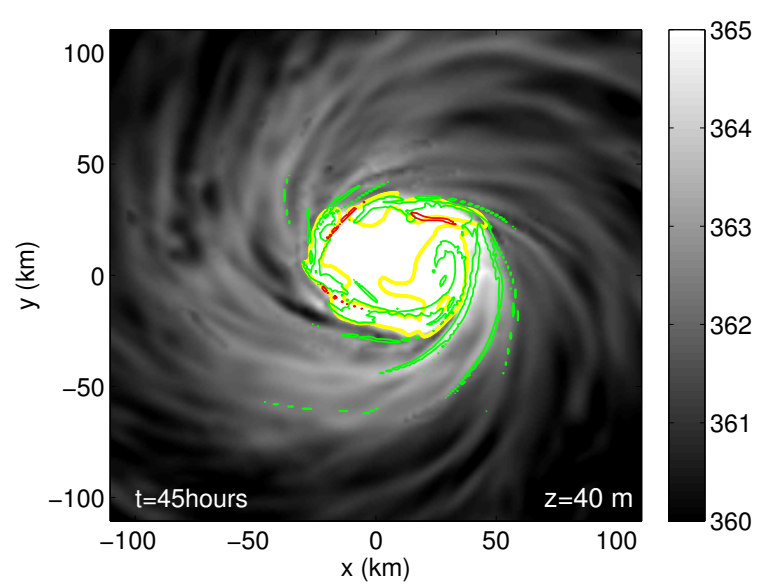

(d)

Fig. 16. The primary Lagrangian structures present during primary eyewall formation at the bottom of the boundary layer as described in Sect. 4.6 are labeled by arrows and are shown at $30 \mathrm{~h}$ (a), $35 \mathrm{~h}$ (b), $40 \mathrm{~h}$ (c), and $45 \mathrm{~h}$ (d). Attracting $\Psi$-LCSs (green), repelling $\Psi$-LCSs (red), and vorticity contours (yellow) are all overlaid on the $\theta_{\mathrm{e}}$ field (black and white shading).

coherent LCSs at the periphery of the vorticity pools that all persist for at least $6 \mathrm{~h}$.

These LCSs are associated with vortical remnants that remain intact for much longer than the normal $1 \mathrm{~h}$ lifetime of a VHT, and grow to diameters of $30 \mathrm{~km}$. These vortices are involved in many organized mergers as described in Sect. 4.5 along persistent $\boldsymbol{\Psi}$-LCSs. Outside organized regions, the LCSs are generally not coherent for longer than $1 \mathrm{~h}$, and the VHTs do not leave behind persistent remnants.

Organized regimes as defined above are approximately outlined by boxes, and labeled "O". At $20 \mathrm{~h}$, there are two primary vortical remnants, seen as yellow circles, residing in $\mathrm{O} 1$ and $\mathrm{O} 2$. Both the vortical remnants and the $\boldsymbol{\Psi}$-LCSs found at $20 \mathrm{~h}$ can be tracked and are still present at $30 \mathrm{~h}$. The $\boldsymbol{\Psi}$-LCSs travel with the vortices and show that the vortices remain separated from turbulence. An additional organized region $\mathrm{O} 3$ emerges at approximately $26 \mathrm{~h}$ and can be tracked beyond $30 \mathrm{~h}$.

\subsection{Formation of primary eyewall}

The primary eyewall formation has started by $30 \mathrm{~h}$, and is shown from $30 \mathrm{~h}$ to $45 \mathrm{~h}$ in Fig. 16. By $30 \mathrm{~h}$, there is a notable reduction in the number of VHTs in the boundary layer. The decrease is also coincident with a decrease in the number of VHTs. Some of the larger remnant pools of vorticity tend to last longer at this time, and are associated with LCSs that also persist for longer than $1 \mathrm{~h}$. Until $35 \mathrm{~h}$, the $\theta_{\mathrm{e}}$ profile remains asymmetric, and long LCSs span the region of enhanced $\theta_{\mathrm{e}}$ and connect the regions of high vorticity. By $40 \mathrm{~h}$, the $\theta_{\mathrm{e}}$ profile is symmetric and the vorticity pools are stretched into a largely ring-like structure. The LCSs merge and form into LCSs whose lengths are greater than the lengths of the LCSs from $20 \mathrm{~h}$ to $30 \mathrm{~h}$. These LCSs wrap around the region of high vorticity and $\theta_{\mathrm{e}}$ and form a complete enclosure of the enhanced $\theta_{\mathrm{e}}$ by $45 \mathrm{~h}$. 


\section{Conclusions}

Using 3-D Lagrangian fields, we have explored 3-D structural aspects of tropical cyclone intensification employing an idealized numerical experiment from prior work. Though the vertical motion associated with convection within VHTs provides a challenging setting for understanding mixing, the LCSs from our kinematically derived fields are able to isolate the persistent signatures of VHTs. A series of conceptual models for binary vortex interaction in a planar flow help illustrate the role of LCSs near vortices, and the interaction described in the models is shown to persist and can be tracked through turbulent flow. Organized flow regimes indicate that the flow structure near the persistent vortical remnants is far different than near convectively active VHTs. The LCSs were shown to complement the system-scale inflow of the boundary layer by highlighting pathways for the stirring of $\theta_{\mathrm{e}}$ and vorticity along attracting LCSs. Above the boundary layer, where the mean inflow is not as dominant, repelling LCSs emerged in conjunction with attracting LCSs as the stirring of the vortical remnants occurred while flow boundaries protect them from the resolved turbulence. The LCSs showed vertical coherence along with the vortices, and a trajectory analysis showed that convection was marked by a tangle of FTLEs. The influence of the localized LCSs concludes with the formation of the primary eyewall, as the preferred vortical remnants and the LCSs which travel with them are stretched into a ring of vorticity and an enclosed attracting LCS.

Though this study has been conducted using model data calculated from an idealized initialization which represents one member of an ensemble, our 3-D Lagrangian methods resulted in 3-D continuous LCSs persisting over varying initial time, and should provide similar results for any of the ensemble members associated with small perturbations in boundary layer moisture as described in Nguyen et al. (2008). The Lagrangian methods used in this study are applicable to a variety of time-dependent vortex flows from small scale turbulence to the system-scale circulation. Vortex behavior on these differing spatial scales can be studied by changing the integration time of the Lagrangian fields. These methods may uncover important localized aspects of transport that have been unattainable by other methods such as eddy statistics. The local dynamics associated with VHTs and aspects of vortex-tube stretching will be pursued in a further study.

Acknowledgements. This work was supported by the National Science Foundation under NSF Cooperative Agreement AGS-0715426 and the support of NSF AGS-0733380, NOAA's Hurricane Research Division and NASA grants NNH09AK561, NNG11PK021 and NNG09HG031. The authors thank Wayne Schubert, Michael Kirby, John Persing, and Michael Riemer for their discussions.

Edited by: T. J. Dunkerton

\section{References}

Bell, M. and Montgomery, M. T.: Sheared deep vortical convection in pre-depression Hagupit during TCS08, Geophys. Res. Lett., 37, L06802, doi:10.1029/2009GL042313, 2010.

Bolton, D.: The computation of equivalent potential temperature, Mon. Weather Rev., 108, 1046-1053, 1980.

Bui, H. H., Smith, R. K., Montgomery, M. T., and Peng, J.: Balanced and unbalanced aspects of tropical cyclone intensification, Q. J. Roy. Meteorol. Soc., 135, 1715-1731 2009.

Chen, Y. and Yau, M. K.: Asymmetric structures in a simulated landfalling hurricane, J. Atmos. Sci., 60, 2294-2312, 2003.

Chen, Y., Brunet, G., and Yau, M. K.: Spiral bands in a simulated hurricane part II: wave activity diagnostics, J. Atmos. Sci., 60, 1239-1256, 2003.

Cohen, R. A. and Schultz, D. M.: Contraction rate and its relation to frontogenesis, the Lyapunov exponent, fluid trapping, and airstream boundaries, Mon. Weather Rev., 133, 1353-1369, 2005.

d'Ovidio, F., Isern-Fontanet, J., Lopez, C., Hernndez-Garca, E., and Garca-Ladona, E.: Comparison between Eulerian diagnostics and finite-size Lyapunov exponents computed from altimetry in the Algerian basin, Deep Sea Res. Part I: Oceanographic Research Papers, 56, 15-31, 1993.

Dudhia, J.: A non-hydrostatic version of the penn state-ncar mesoscale model: Validation tests and simulation of an atlantic cyclone and cold front, Mon. Wheather Rev., 8, 1493-1513, 1993.

Dunkerton, T. J., Montgomery, M. T., and Wang, Z.: Tropical cyclogenesis in a tropical wave critical layer: easterly waves, Atmos. Chem. Phys., 9, 5587-5646, doi:10.5194/acp-9-5587-2009, 2009.

Emanuel, K. A., Neelin, J. D., and Bretherton, C. S.: On large scale circulations in convecting atmosphere, Q. J. Roy. Meteorol. Soc., 120, 1111-1143, 1994.

Fang, J. and Zhang, F.: Initial development and genesis of Hurricane Dolly (2008), J. Atmos. Sci., 67, 655-672, 2010.

Grell, G., Dudhia, J., and Stauffer, D.: A description of the fifth generation Penn State-NCAR Mesoscale Model (MM5), NCAR Tech. Note NCAR/TN, 398, 1-138, 1995.

Haller, G.: Finding finite-time invariant manifolds in twodimensional velocity fields, Chaos, 10, 99-108, 2000.

Haller, G.: Lagrangian coherent structures from approximate velocity data, Phys. Fluids, 14, 1851-1861, 2002.

Haller, G. and Iacono, R.: Stretching, alignment, and shear in slowly varying velocity fields, Physical Review E, 68, 056304-1-6, 2003.

Haller, G. and Poje, A.: Finite time transport in aperiodic flows, Physica D, 119, 352-380, 1997.

Haller, G. and Yuan, G.: Lagrangian coherent structures and mixing in two-dimensional turbulence, Physica D, 147, 352-370, 2000.

Hendricks, E. A., Montgomery, M. T., and Davis, C. A.: On the role of vortical hot towers in tropical cyclone formation, J. Atmos. Sci., 61, 1209-1232, 2004.

Holton, J. R.: An introduction to dynamic meteorology, 4th edition, Elsevier, 2004.

Ide, K., Small, D., and Wiggins, S.: Distinguished hyperbolic trajectories in time-dependent fluid flows: analytical and computational approach for velocity fields defined as data sets, Nonlin. Processes Geophys., 9, 237-263, doi:10.5194/npg-9-237-2002, 
2002.

Joseph, B. and Legras, B.: Relation between Kinematic Boundaries, Stirring, and Barriers for the Antarctic Polar Vortex, J. Atmos. Sci., 59, 1198-1212, 2002.

Lapeyre, G.: Characterization of finite-time Lyapunov exponents and vectors in two-dimensional turbulence, Chaos, 12, 688-698, 2002.

Levina, G. and Montgomery, M. T.: A first examination of the helical nature of tropical cyclogenesis, Doklady Earth Sciences, 434, Part I, 1285-1289, 2010.

Lilly, D. K.: On the theory of disturbances in a conditionally unstable atmosphere, Mon. Weather Rev., 88, 1-17, 1960.

Malhotra, N. and Wiggins, S.: Geometric structures, lobe dynamics, and Lagrangian transport in flows with aperiodic timedependence, with applications to Rossby wave flow, J. Nonlinear Sci., 8, 401-456, 1999.

Melander, N. J., Zabusky, M. V., and McWilliams, J. C.: Symmetric vortex merger in two dimensions - causes and conditions, J. Fluid Mech., 195, 303-340, 1988.

Montgomery, M. T. and Smith, R. K.: Paradigms for tropicalcyclone intensification, Q. J. Roy. Meteorol. Soc., in review, 2012.

Montgomery, M. T., Nichols, M. E., Cram, T. A., and Saunders, A. B.: A vortical hot tower route to tropical cyclogenesis, J. Atmos. Sci., 63, 355-386, 2006.

Montgomery, M. T., Nguyen, V. S., Smith, R. K., and Persing, J.: Do tropical cyclones intensify by WISHE?, Q. J. Roy. Meteorol. Soc., 135, 1697-1714, 2009.

Montgomery, M. T., Wang, Z., and Dunkerton, T. J.: Coarse, intermediate and high resolution numerical simulations of the transition of a tropical wave critical layer to a tropical storm, Atmos. Chem. Phys., 10, 10803-10827, doi:10.5194/acp-1010803-2010, 2010.

Ngan, K. and Shepherd, T. G.: A Closer Look at Chaotic Advection in the Stratosphere. Part I: Geometric Structure, J. Atmos. Sci., 56, 4134-4152, 1999.

Nguyen, S. A., Smith, R. K., and Montgomery, M. T.: Tropicalcyclone intensification and predictability in three dimensions, Q. J. Roy. Meterol. Soc., 134, 563-582, 2008.

Nguyen, C. M., Reeder, M. J., Davidson, N. E., Smith, R. K., and Montgomery, M. T.: Inner-core vacillation cycles during the rapid intensification of Hurricane Katrina, Q. J. Roy. Meteorol. Soc., 137, 829-844, 2011.

Ottino, J. M.: The kinematics of mixing: stretching, chaos, and transport, Annual Reviews of Fluid Mechanics, 22, 207-53, 1990.

Pierrehumbert, R. T.: Large-scale horizontal mixing in planetary atmospheres, Phys. Fluids A, 3, 1250-1260, doi:10.1063/1.858053, 1991.

Pierrehumbert, R. T. and Yang, H.: Global chaotic mixing on isentropic surfaces, J. Atmos. Sci., 50, 2462-2480, 1993.

Prieto, R., McNoldy, B. D., Fulton, S. R., and Schubert, W. H.: A classification of binary tropical-cyclone-like vortex interactions, Mon. Weather. Rev., 131, 2656-2666, 2003.

Provenzale, A.: Transport by coherent barotropic vortices, Annual Review of Fluid Mechanics, 31, 55-93, 1999.

Raymond, D. J. and López Carrillo, C.: The vorticity budget of developing typhoon Nuri (2008), Atmos. Chem. Phys., 11, 147163, doi:10.5194/acp-11-147-2011, 2011.
Reasor, P. D., Montgomery, M. T., and Bosart, L.: Mesoscale observations of the genesis of Hurricane Dolly (1996), J. Atmos. Sci., 62, 3151-3171, 2005.

Rutherford, B. and Dangelmayr, G.: A 3d Lagrangian hurricane eyeeyewall computation, Q. J. Roy. Meteorol. Soc., 136, 1931-1944, 2010.

Rutherford, B., Dangelmayr, G., Persing, J., Kirby, M., and Montgomery, M. T.: Lagrangian mixing in an axisymmetric hurricane model, Atmos. Chem. Phys., 10, 6777-6791, doi:10.5194/acp10-6777-2010, 2010a.

Rutherford, B., Dangelmayr, G., Persing, J., Schubert, W. H., and Montgomery, M. T.: Advective mixing in a nondivergent barotropic hurricane model, Atmos. Chem. Phys., 10, 475-497, doi:10.5194/acp-10-475-2010, 2010b.

Saffman, P. G.: Dynamics of vorticity, J. Fluid Mechanics, 106, 4958,1981 .

Sapsis, T. and Haller, G.: Inertial particle dynamics in hurricanes, J. Atmos. Sci., 66, 2481-2492, 2009.

Schubert, W. H. and Hack, J. J.: Inertial stability and tropical cyclone development, J. Atmos. Sci., 39, 1687-1697, 1982.

Scott, R. K., Shuckburgh, E. F., Cammas, J. P., and Legras, B.: Stretching rates and equivalent length near the tropopause, J. Geophys. Res., 108, 4394, doi:10.1029/2002JD002988, 2003.

Shadden, S.: A dynamical systems approach to unsteady flows, Ph.D. thesis, California Institute of Technology, 2006.

Shadden, S. C., Lekien, F., and Marsden, J. E.: Definition and properties of Lagrangian coherent structures from finite-time Lyapunov exponents in two-dimensional aperiodic flows, Physica D, 212, 271-304, 2005.

Shadden, S. C., Dabiri, J. O., and Marsden, J. E.: Lagrangian analysis of fluid transport in empirical vortex rings, Phys. Fluids, 18, 047105-1-11, 2006.

Shin, S. and Smith, R. K.: Tropical cyclone intensification and predictability in a minimal three-dimensional model, Q. J. Roy. Meteorol. Soc., 134, 1385-1395, 2008.

Siggia, E.: High Raleigh number convection, Annu. Rev. Fluid Mech., 26, 137-168, 1994.

Sippel, J. A., Nielsen-Gammon, J. W., and Allen, S. E.: The multiple- vortex nature of tropical cyclogenesis, Mon. Weather Rev., 134, 1796-1814, 2006.

Smith, R. K., Montgomery, M. T., and Vogl, S.: A critique of Emanuel's hurricane model and potential intensity theory, Q. J. Roy. Meteorol. Soc., 134-632, 551-561, 2008.

Smith, R. K., Montgomery, M. T., and Nguyen, V. S.: Tropical cyclone spin up revisited, Q. J. Roy. Meteorol. Soc., 134, 13851395, 2009.

Tang, W., Mathur, M., Haller, G., Hahn, D. C., and Ruggiero, F. H.: Lagrangian coherent structures near a subtropical jet stream, J. Atmos. Sci., 67, 2307-2319, 2010.

Truesdell, C.: The kinematics of vorticity, Indiana Uiversity Press, 1954.

von Hardenberg, J., Mc Williams, J. C., Provenzale, A., Shchepetkin, A., and Weiss, J. B.: Vortex merging in quasi-geostrophic flows, J. Fluid Mech., 412, 331-353, 2000.

Velasco Fuentes, O.: Chaotic advection by two interacting finitearea vortices, Phys. of Fluids, 13-4, 901-912, 2001.

Wang, Y.: Vortex rossby waves in a numerically simulated tropical cyclone. part ii: The role in tropical cyclone structure and intensity changes, J. Atmos. Sci., 59, 1239-1262, 2000. 
Weiss, J. B. and Provenzale, A.: Transport and mixing in geophysical flows, Springer, 2008.

Wiggins, S.: The dynamical systems approach to Lagrangian transport in oceanic flows, Annual Review of Fluid Mechanics, 37, 295-328, 2005.
Willoughby, H. E.: Forced secondary circulations in hurricanes, J. Geophys. Res., 84, 3173-3183, 1979. 